QUARTERLY OF APPLIED MATHEMATICS

VOLUME LXIX, NUMBER 3

SEPTEMBER 2011, PAGES 569-601

S 0033-569X(2011)01226-X

Article electronically published on May 9, 2011

\title{
LOSS OF CONTROL OF MOTIONS FROM INITIAL DATA FOR PENDING CAPILLARY LIQUID
}

\author{
BY \\ UMBERTO MASSARI (Department of Mathematics, University of Ferrara, Via Machiavelli 35, \\ 44100 Ferrara, Italy), \\ MARIAROSARIA PADULA (Department of Mathematics, University of Ferrara, Via Machiavelli \\ 35, 44100 Ferrara, Italy), \\ AND \\ SENJO SHIMIZU (Faculty of Science, Shizuoka University, Ohya 836, Shizuoka 422-8529, Japan)
}

Abstract. First, the problem of stability of an equilibrium figure $F_{*}$ for an abstract system is reduced to the sign of the difference between the energy of the perturbed motion at initial time, and that of $F_{*}$. All control conditions are only sufficient conditions to ensure nonlinear stability.

Second, employing the local character of the nonlinear stability, some nonlinear instability theorems are proven by a direct method.

Third, the definition of loss of control from initial data for motions $F$ is introduced. A class of equilibrium figures $F_{*}$ is constructed such that: $F_{*}$ is nonlinearly stable; the motions, corresponding to initial data sufficiently far from $F_{*}$, cannot be controlled by their initial data for all time. A lower bound is computed for the norms of initial data above which the loss of control from initial data occurs.

1. Introduction. Let a capillary liquid drop $F$ be pending below a rigid surface $\mathcal{S}$ with air at rest all around. We denote its equilibrium position by $F_{*}$. Usually a blast perturbs $F$ that changes its position from $F_{*}$ into $F_{0}$. If the blast is sufficiently light, $F_{0}$ will be sufficiently close to $F_{*}$ and possibly $F$ will oscillate around $F_{*}$. On the contrary, if the blast is sufficiently intense, then $F_{0}$ will be sufficiently far from $F_{*}$, at the initial time $t=0$, and possibly at a subsequent time $t>0, F$ will follow down. We address the following equivalent questions:

Received March 30, 2010.

2000 Mathematics Subject Classification. Primary 35Q30, 76D05.

Key words and phrases. Nonlinear instability, Rayleigh-Taylor instability, horizontal layer flow, free boundary problem, Navier-Stokes equation, surface tension, gravity force.

E-mail address: umberto.massari@unife.it

E-mail address: pad@unife.it

E-mail address: ssshimi@ipc.shizuoka.ac.jp 
Question $\mathbf{Q}_{1}$. Is it possible to compute a lower bound on the blast's intensity such that the liquid motion $F$, corresponding to the blast's perturbation as initial data, represents the detachment and the fall down of a liquid drop?

Question $\mathbf{Q}_{2}$. Is it possible to compute a lower bound on the geometrical measure of $F_{0}$ such that the liquid drop $F$, in correspondence to $F_{0}$ as initial data, will fall down at a certain time $t$ ?

Such questions arise when the rest state is linearly stable, and therefore the rest state is also nonlinearly stable for sufficiently small initial data.

However, in reality, the initial data are large; thus the information on linear stability is in general not sufficient to ensure that the rest state will be observed. In this paper we look for initial data which are far from a stable rest state.

Our question focalizes essentially on the nonlinear aspect of the physical world; namely, we focus our attention on one of the central problems of fluid mechanics: when and how laminar flows break down? Our results should be considered as a first contribution to give an answer to such a question.

Even though the question appears elementary, until now we have not found a correct mathematical position for such a question; furthermore, the mathematical formulation of such a problem that we are addressing here seems to be not so trivial. Therefore in this paper we limit ourselves to the mathematical formulation of problem $\mathbf{Q}_{2}$ when instead of a liquid pending drop we have a section of a liquid layer, so that there are no contact angles, and the geometry is the Cartesian one.

One example. Let $G$ denote a pure number used as a stability parameter. For us $G$ is the Grashof number for a heavy layer of liquid motion, and it is a linear function of the gravity intensity, and an inverse function of the surface tension and of the area of rigid surface. It is known that a capillary liquid layer is linearly stable for all $G$ if the rigid plane is situated below the liquid layer. Here it is proved that the capillary liquid layer, if the rigid plane is situated above the liquid layer, is still linearly stable for all $G$ sufficiently small, say if $G<G_{d}$, where $G_{d}$ is a given number; cf. (6.8).

Main goals. One aim is to reduce the study of stability, linear or not, to the sign of a suitable functional $\mathbb{E}$ called the modified energy functional. Such a goal is achieved providing a direct stability method that reduces the stability problem to computation of a critical number, say $G_{d}$, below which the motion is nonlinearly stable for sufficiently small initial data.

Another aim is to study the control of a motion $F$ in terms of initial data $F_{0}$, when $F_{0}$ is sufficiently far from the equilibrium figure $F_{*}$.

REMARK 1.1. We remark that the method here proposed doesn't require the knowledge of the solution; moreover, all computations are relatively simple because they are reduced to the sign of a well-known functional. Here we give a pure nonlinear method to compute: i) nonlinear stability of the equilibrium configuration $F_{*}$ sufficiently small; ii) loss of control of the equilibrium configuration $F_{*}$ if the initial data of perturbed motion are sufficiently large; iii) linear instability of a liquid layer with free boundary below the liquid layer. The problem of linear stability has recently received the attention of several writers [26, 27, 28. Concerning instability, there is a partial answer in [21], [19, 36]. 
Why it may be interesting. We prove nonlinear stability, or instability only for initial data sufficiently small, say in the ball $B_{\delta}$. The radius $\delta$ goes to zero as $G$ increases toward $G_{d}$, and the initial data become no longer controllable because it is impossible to choose initial data smaller than a given value $\delta$ if it is "too small".

Let us now give some earlier results of well posedness. Here we have distinguished the references into two parts. The first set of articles is more related to existence and stability problems, while the second set of references is more directed toward instability questions.

The motion of a horizontal capillary layer of viscous, incompressible fluid bounded below by a rigid surface and above by a free surface, taking surface tension into account, has been studied by several authors. Existence theorems for such problem have been proved by Beale [3, Beale and Nishida [4, Allain [2, Tani [31, Sylvester [30], Tani and Tanaka [32, Abels [1, Nishida, Teramoto and Yoshihara [14, Teramoto [33, 34, Nishida, Teramoto and Win [16, Shibata and Shimizu [29], Prüss and Simonett [22], Padula and Solonnikov [37] for incompressible fluids. Jin and Padula [10, 11] proved it for compressible fluids.

The inverse situation where the rigid boundary is above the liquid layer allows vertical disturbance in the velocity in the vertical direction $x_{3}$ that may produce novel features of instability, so-called Rayleigh-Taylor instability. The Rayleigh-Taylor stability of the problem has been studied by Chandrasekhar [5], Drazin and Reid [6], Whitehead and Chen [35, Helffer and Lafitte [12, Nishida, Teramoto and Yoshihara [15], Iooss and Rossi [13. All the above results are proved in connection with small initial data. It is well known that a stability threshold computed by linear theory does not ensure nonlinear stability for large initial data; i.e., the stability thresholds computed by nonlinear approach will not coincide with stability thresholds computed by linear theory. A basic flow judged linearly stable could be found to be unobservable for noninfinitesimal initial perturbations. Therefore it makes sense to study nonlinear stability or instability directly without a linearized procedure as in Padula [19, Padula and Solonnikov [20, 21] and Frolova and Padula [8, 36].

We now give the theme of the paper. We devote the first two sections to formulating correctly the mathematical problem for a section of a layer. Precisely in section 2 we introduce the definitions of Equilibrium Figure used in Capillarity Theory, of Rest State and of Perturbation to the Rest State used in fluid dynamics with free boundary $*$ Since our mathematical model involves several fields of analysis such as Variational Methods, Partial Differential Equations, Stability Methods, sometimes we also write, or prove, explicitly some standard formulas. In section 3 we formulate the problem and we give the main theorems. Essentially we use a notion that we name control from initial data that is similar to that of continuous dependence on the data in the time interval $(0, T)$, the only difference lying in the fact that the time interval is $(0, \infty)$. In other words, the control from initial data in $\mathcal{I}_{a, 2 a}$ is a uniform continuity, in a given norm, of motions corresponding to initial data in $\mathcal{I}_{a, 2 a}$. In section 4 we prove the main theorems concerning nonlinear stability, and instability results $\dagger$ and we compute a lower bound to initial data

\footnotetext{
${ }^{*}$ Equilibrium figures and rest state are different concepts.

$\dagger$ The proofs can be extended to more general domains.
} 
above which the liquid layer falls down. Furthermore in section 5 we prove asymptotic stability results; to this end, using the free work identity, we construct a modified energy functional. In section 6 for a heavy layer of fluid, with upper rigid boundary and lower free boundary, we construct an example of a rest state $S_{b}$, within a given parallelepiped $F_{*}$ of volume $V=\left|\Omega_{b}\right|=h|\Sigma|$, where $\Sigma$ is the wet area, and with given surface tension $\sigma$. Such a figure $F_{*}$ is linearly stable if the Grashoff number $G=g|\Sigma| / \sigma$, where $g$ is the gravity, is sufficiently small: $G<G_{d}$. Moreover for large initial data, in correspondence to the same Grashoff number, provided the shape of the pending drop is suitablet the $L^{2}$-norm of the motion loses its control from the initial data. We end the paper with an appendix on linear stability methods.

We conclude the Introduction with a remark.

Denote by $S\left(S_{0}, t\right)$ the motion of the liquid section of the layer corresponding to the initial data $S_{0}$. Assume that the function $S\left(S_{b}, t\right)=S_{b}$ is an exact stationary solution of the liquid layer, corresponding to the initial data $S_{b}$. In this paper we reduce the problem of stability, linear or not, to the sign of the given "energy of perturbation"

$$
\mathcal{E}\left(S\left(S_{0}, t\right)\right):=E\left(S_{0}\right)-E\left(S_{b}\right)
$$

where $E\left(S\left(S_{0}\right)\right)$ denotes the total energy of the physical system, at time $t$, corresponding to the initial data $S_{0}$. We furnish the above proofs through Lyapunov direct methods. Our main tool consists in the introduction of a new functional by using the idea of a free work identity, which was established by one of the authors, Padula [17, 18, 19]. It is worth emphasizing that our method is simple, straightforward, and does not use complicated analysis.

The authors thank the referee for deep remarks that have substantially improved the paper.

2. Equilibrium figures, Free BVP. In this section we introduce some known results on equilibrium figures and liquid motions above or below a horizontal rigid layer.

2.1. Equilibrium figures. Concerning equilibrium figures of viscous incompressible capillary fluids we like mentioning two capillary prototypes: a self-gravitating liquid drop; a section of a horizontal layer of heavy liquid. In capillarity theory the unknown domain $\Omega_{*}$ occupied by the liquid, referred to as the equilibrium figure, is defined by the equation of its boundary $\partial \Omega_{*}$. Here we consider the boundary $\partial \Omega_{*}$ as the union of several surfaces, namely $\partial \Omega_{*}=\Gamma_{*} \cup \Gamma_{B} \cup \Gamma_{l}$, with $\Gamma_{B}$ the rigid part of the boundary $\partial \Omega_{*}, \Gamma_{*}$ the deformable part of the boundary $\partial \Omega_{*}$, called a free surface, and $\Gamma_{l}$ the fixed geometrical part of $\partial \Omega_{*}$. In both of our examples it is $\overline{\Gamma_{*}} \cap \overline{\Gamma_{B}}=\emptyset$; namely, we do not consider contact angles. This implies that either $\Gamma_{B}=\emptyset$ (liquid drop) and thus we have $\Gamma_{l}=\emptyset$, or $\Gamma_{B} \neq \emptyset$ (layer) and thus $\Gamma_{l} \neq \emptyset$ will be the union of two parts, $\Gamma_{l}=\Gamma_{1} \cup \Gamma_{2}$, where we prescribe periodicity conditions.

In the sequel, we consider a horizontal layer of heavy viscous fluid bounded above or below, respectively, by a rigid surface $\Gamma_{B}$ and below or above, respectively, by a free

\footnotetext{
¥A typical shape of a pending liquid layer, where there is loss of control from initial data, is realized by taking the wet area $|\Sigma|$ small compared to the thickness $h$ of the layer.
} 
surface $\Gamma_{t}$. Let us introduce a Cartesian reference frame $\mathcal{R}=\left\{O, \mathbf{e}_{1}, \mathbf{e}_{2}, \mathbf{e}_{3}\right\}$ with $\mathbf{e}_{3}$ vertical, and let $x_{1}, x_{2}, x_{3}$ be the coordinate axes. The orientation of the vertical axis $x_{3}$ is done in the direction of the free surface $\Gamma_{t}$ upward or downward according as $\Gamma_{B}$ is situated below or above the free surface $\Gamma_{t}$, respectively $\$$ Also $x^{\prime}=\left(x_{1}, x_{2}\right)$ denote the components of $x$ in the periodicity cell $\Sigma=(0,1) \times(0, d) \subset \mathbb{R}^{2}$. Here we take $\Sigma$ such that the constant of the Poincaré inequality

$$
\|\eta\|_{L^{2}(\Sigma)}^{2} \leq C_{d}\left\|\nabla^{\prime} \eta\right\|_{L^{2}(\Sigma)}^{2}
$$

is equal to $C_{d}^{-1}=\lambda_{1}=: 4 \pi^{2} \min \left\{1,1 / d^{2}\right\}$. We adopt the Cartesian representation both for a rigid surface $\Gamma_{B}$ and a free surface $\Gamma_{t}$ :

$$
\begin{gathered}
\Gamma_{B}=\left\{x=\left(x^{\prime}, x_{3}\right) \in \mathbb{R}^{3}: x^{\prime} \in \Sigma, x_{3}=f\left(x^{\prime}\right)\right\}, \\
\Gamma_{t}=\left\{x=\left(x^{\prime}, x_{3}\right) \in \mathbb{R}^{3}: x^{\prime} \in \Sigma, x_{3}=h+\eta\left(x^{\prime}, t\right)\right\},
\end{gathered}
$$

where $f\left(x^{\prime}\right)$ is a given function. In order to have a simply connected domain for all time, we must assume that

$$
h+\eta\left(x^{\prime}, t\right)>f\left(x^{\prime}\right) \quad \text { for all } t \geq 0 .
$$

Let a massic potential $U$ be given, and let $\mathcal{H}$ be the curvature of a surface $\Gamma$. The equation of the free surface $\Gamma_{*}$ is given as a solution $\zeta=\zeta_{*}\left(x^{\prime}\right)$ to the elliptic, LaplaceBeltrami equation

$$
\sigma \mathcal{H}(\zeta)+U(\zeta)+q_{e}=0, \quad x^{\prime} \in \Sigma,
$$

with $\sigma$ the surface tension divided by a uniform density, and $q_{e}$ the external constant pressure divided by a uniform density. The solutions $\zeta=\zeta_{*}$ to (2.3) denote the equilibrium figures.

We recall that solutions to (2.3) provide the stationary points of the nonlinear functional, representing the total energy at rest,

$$
E_{U}=\sigma(|\Gamma|-|\Sigma|)-\int_{\Omega} U d x-q_{e}|\Omega|
$$

here |.| denotes the measure of the sets $\Gamma, \Omega$. The nonlinear functional $E_{U}$ is considered in the set of domains $\Omega$ close to $\Omega_{*}$, with the same volume and the position of the barycenter coinciding with that of $\Omega_{*}$, with $\partial \Omega=\Gamma \cup \Gamma_{B} \cup \Gamma_{l}, \partial \Omega_{*}=\Gamma_{*} \cup \Gamma_{B} \cup \Gamma_{l}$, and $\Gamma$ close to $\Gamma_{*}$.

Notice that the sign of $E_{U}$ is a function of the external potential that may be gravitational, centrifugal, etc.; indeed, the external forces rule the stability of an equilibrium figure.

To be more clear, we make explicit computations in the simplified case of a layer, $\Gamma_{l} \neq 0$. Let $\zeta_{i}$ denote the partial derivative of $\zeta$ with respect to $x_{i}, \zeta_{i}=\frac{\partial \zeta}{\partial x_{i}}$, and in the case of a layer we may we write

$$
E_{U}(\zeta)=\mathcal{F}\left(\zeta, \zeta_{i}\right):=\int_{\Sigma}\left(F\left(\zeta_{i}\right)+f(\zeta)\right) d x
$$

\footnotetext{
$\S$ We assume that the liquid is below the rigid plane $\Gamma_{B}$ because we need negative potential energy. We notice that a negative potential energy can be simply realized by rapidly accelerating a beaker of water downwards (and standing clear!).
} 
The Fréchet derivative of $\mathcal{F}$ in $\zeta_{*}$ is given by

$\delta \mathcal{F}\left(\zeta_{*}, \zeta_{* i}\right)\left[\zeta, \zeta_{i}\right]=\left.\frac{d}{d t} \int_{\Sigma}\left(F\left(\zeta_{* i}+t \zeta_{i}\right)+f\left(\zeta_{*}+t \zeta\right)\right) d x\right|_{t=0}=\int_{\Sigma}\left(\sum_{i} \frac{\partial F}{\partial \zeta_{i}}\left(\zeta_{* i}\right) \zeta_{i}+f^{\prime}\left(\zeta_{*}\right) \zeta\right) d x$

where

$$
f^{\prime}\left(\zeta_{*}\right):=\left.\frac{d f}{d \zeta}\right|_{\zeta=\zeta_{*}}
$$

Equation (2.3) is the Euler-Lagrange equation associated to $E_{U}$, and with our notation we get

$$
-\sum_{i} \frac{\partial}{\partial x_{i}} \frac{\partial F}{\partial \zeta_{i}}\left(\zeta_{* i}\right)+f^{\prime}\left(\zeta_{*}\right)=0
$$

Formally (2.3), and (2.6) in the simplified case of a layer, can be represented by means of the nonlinear operator $B_{U}$ as the equation

$$
B_{U}\left(\zeta, \zeta_{i}\right)=-\sigma \Delta_{\Gamma} \zeta(x, t)+b(x) \zeta(x, t)+q_{e}=0,
$$

where

$$
-\Delta_{\Gamma}(\zeta)=-\sum_{i} \frac{\partial}{\partial x_{i}} \frac{\partial F\left(\zeta_{i}\right)}{\partial \zeta_{i}}
$$

is the nonlinear positive Laplace-Beltrami operator on $\Gamma$, and

$$
b(x)=\frac{f^{\prime}(\zeta)}{\zeta}-\frac{q_{e}}{\zeta}
$$

is related to the potential $U$; see [21, 223, 24]-28]

In conclusion by (2.5), (2.6) is equivalent to (2.7) and, in the difference $\eta=\zeta-\zeta_{*}$ we get

$$
\delta \mathcal{F}\left(\zeta_{*}, \zeta_{* i}\right)\left[\eta, \eta_{i}\right]=\delta E_{U}\left(\zeta_{*}\right)[\eta]=0
$$

The second variation of $E_{U}$, computed in $\zeta_{*}$, is a quadratic form in the difference $\eta=$ $\zeta-\zeta_{*}:$

$$
\begin{aligned}
& \delta^{2} E_{U}\left(\zeta_{*}\right)\left[\zeta, \zeta_{i}\right]=\left.\frac{d}{d t} \int_{\Sigma}\left(\left.\sum_{i} \frac{\partial F\left(\zeta_{i}\right)}{\partial \zeta_{i}}\right|_{\zeta_{* i}+t \zeta_{i}} \eta_{i}+\left.f^{\prime}(\zeta)\right|_{\zeta_{*}+t \zeta} \eta\right) d x\right|_{t=0} \\
& =\int_{\Sigma}\left(\left.\sum_{i} \frac{\partial^{2} F\left(\zeta_{i}\right)}{\partial \zeta_{i}^{2}}\right|_{\zeta_{*}} \eta_{i}^{2}+\left.f^{\prime \prime}(\zeta)\right|_{\zeta_{*}} \eta^{2}\right) d x .
\end{aligned}
$$

On the other side we have

$$
\begin{aligned}
\delta \mathcal{F}\left(\zeta_{*}\right. & \left.+t \zeta, \zeta_{* i}+t \zeta_{i}\right)[\eta]=\frac{d}{d t} \delta \mathcal{F}\left(\zeta_{*}+t \zeta, \zeta_{* i}+t \zeta_{i}\right)\left[\eta, \eta_{i}\right]+o\left(\eta^{2},, \eta_{i}^{2}\right) \\
& =\left.\frac{d}{d t} \int_{\Sigma}\left(\left.\sum_{i} \frac{\partial F\left(\zeta_{i}\right)}{\partial \zeta_{i}}\right|_{\zeta_{*}+t \zeta, \zeta_{* i}+t \zeta_{i}} \eta_{i}+\left.f^{\prime}(\zeta)\right|_{\zeta_{*}+t \zeta} \eta\right) d x\right|_{t=0}+o\left(\eta^{2}, \eta_{i}^{2}\right) \\
& =\delta^{2} E_{U}\left(\zeta_{*}, \zeta_{* i}\right)\left[\eta, \eta_{i}\right]+o\left(\eta^{2}, \eta_{i}^{2}\right) .
\end{aligned}
$$

I We remark that in the case of a drop,

$$
E_{U}(\zeta)=\mathcal{F}\left(\zeta, \zeta_{i}\right):=\int_{\Sigma}\left(F\left(\zeta, \zeta_{i}\right)+f(\zeta)\right) d x
$$

and computations are more involved. In particular, $b(x)$ also involves the surface's terms. 
Subtracting $B_{U}\left(\zeta_{*}, \zeta_{* i}\right)$ from $B_{U}\left(\zeta, \zeta_{i}\right)$ we may write

$$
B_{U}\left(\zeta, \zeta_{i}\right)-B_{U}\left(\zeta_{*}, \zeta_{* i}\right)=: B_{1}\left(\eta, \eta_{i}\right)=\mathcal{L}(\eta)+R\left(\eta, \eta_{i}\right), \quad \eta=\zeta-\zeta_{*}
$$

where we have distinguished the linear part $\mathcal{L}(\eta)$ from the differential nonlinear operator of order higher than one, $R\left(\eta, \eta_{i}\right)=o\left(\eta, \eta_{i}\right)$, and we have set

$$
\mathcal{L}(\eta)=-\sigma \Delta_{\Gamma_{*}} \eta+b(x) \eta
$$

where $\Delta_{\Gamma_{*}}$ is the Laplace-Beltrami operator computed along a given geometry.

Let $(.,$.$) be the L^{2}$ scalar product on $\Gamma$. The functionals

$$
\begin{aligned}
& \left(B_{1}\left(\eta, \eta_{i}\right), \eta\right)=(\mathcal{L}(\eta), \eta)+\left(R\left(\eta, \eta_{i}\right), \eta\right)=\delta^{2} E_{U}\left(\zeta_{*}\right)[\eta], \\
& (\mathcal{L}(\eta), \eta)=\delta^{2} E_{l U}\left(\zeta_{*}\right)[\eta]
\end{aligned}
$$

represent the second variations of the total energy $E_{U}$, and of the quadratic part $E_{l U}$.

Stable equilibrium figure. An equilibrium figure $\mathcal{F}_{*}$ is regarded as globally linearly stable if the second variation $\delta^{2} E_{l U}$ of the quadratic part $E_{l U}$ of the total energy $E_{U}$ is positive and linearly unstable if it can take negative values. The same configuration $\mathcal{F}_{*}$ will also be locally nonlinearly stable, unstable respectively. In other words an equilibrium figure $\mathcal{F}_{*}$ is nonlinearly stable if it is a local minimum for the total energy. Of course, since the energy is defined up to a constant, one can state the previous assertions in a slightly different way: an equilibrium figure is linearly stable if $E_{l U}-E_{*}$ is a positive definite quadratic form, and nonlinearly stable if $E_{U}-E_{*}$ is positive in a neighborhood of the zero solution.

2.2. Initial boundary value problem. Let us begin with two remarks on the stability of capillary figures. First, we consider equilibrium figures, and we study the minimal $\zeta_{*}=\zeta_{*}\left(x^{\prime}, t\right)$ functions of a functional $\mathcal{F}=\mathcal{F}(\zeta)$. Precisely, we deal with perturbations to the $\zeta_{*}$ solution to a scalar equation. Second we furnish a control in time of solutions, which we denote by $S\left(S_{0}, t\right) \equiv(\mathbf{v}, \zeta)$, corresponding to initial data, which we denote by $S_{0} \equiv\left(\mathbf{v}_{0}, \zeta_{0}\right)$, if they are not too far from the exact solutions $S_{b} \equiv\left(\mathbf{0}, \zeta_{\mathbf{b}}\right)$, which in our case represent the rest state. Precisely, given the solution pair $\left(\mathbf{v}_{b}, \zeta_{b}\right)$ to the steady system, we study the evolution in time of solutions $(\mathbf{v}, \zeta)$ obtained by perturbing at the initial time the pair $\left(\mathbf{v}_{b}, \zeta_{b}\right)$. It is clear that the four statements (i),.., (iv) must be rigorously proved; furthermore, we expect that the proof should depend on the equilibrium figure at hand, namely on $G$.

The classical problem of stability of equilibrium figures for a viscous incompressible capillary liquid subjected to potential forces has been the object of high attention in the last fifty years. It consists of the determination of a bounded domain $\Omega_{t} \subset R^{3}$, a velocity vector field $\mathbf{u}(x, t)=\left(u_{1}, u_{2}, u_{3}\right)$, and the scalar pressure (divided by the uniform 
density) $q(x, t), x \in \Omega_{t}, t>0$, satisfying the following relations:

$$
\begin{array}{ll}
\partial_{t} \mathbf{u}+(\mathbf{u} \cdot \nabla) \mathbf{u}-\nabla \cdot T(\mathbf{u}, q)=\nabla U & \text { in } \Omega_{t}, t>0 \\
\nabla \cdot \mathbf{u}=0 & \text { in } \Omega_{t}, t>0 \\
T(\mathbf{u}, q) \mathbf{n}=\sigma \mathcal{H} \mathbf{n}-q_{e} \mathbf{n} & \text { on } \Gamma_{t}, t>0 \\
\mathbf{V} \cdot \mathbf{n}=\mathbf{u} \cdot \mathbf{n} & \text { on } \Gamma_{t}, t>0 \\
\mathbf{u}=\mathbf{0} & \text { on } \Gamma_{B}, t>0 \\
\left.\mathbf{u}\right|_{t=0}=\mathbf{u}_{0} \text { in } \Omega_{0}, \quad \Omega_{0}=\text { given, } &
\end{array}
$$

plus periodicity on $\Gamma_{l}$. Here,

$$
T(\mathbf{u}, q)=\mu S(\mathbf{u})-q I
$$

is the Cauchy stress tensor, $\mu$ the constant kinematical viscosity coefficient,

$$
S(\mathbf{u})=(S(\mathbf{u}))_{i j}=\frac{\partial u_{i}}{\partial x_{j}}+\frac{\partial u_{j}}{\partial x_{i}}
$$

is the deformation tensor, $I$ is the $3 \times 3$ identity matrix, $\mathbf{n}$ is the unit outer normal to $\Gamma_{t}$ and $\mathbf{V}$ is the velocity of points on $\Gamma_{t}$. Also $q_{e}$ is the external pressure, and $\mathcal{H}$ is the doubled mean curvature of $\Gamma_{t}$, which is negative for a convex domain.

A justification of the statement on nonlinear stability has been recently furnished for a layer of fluid [17, 20, and for a drop of liquid [21, 24]-28. Recently for a drop of liquid it has been proved that if $\mathcal{L}$ has at least one negative eigenvalue, then the rest is linearly unstable; see [19], 23].

To explain earlier and present results we deem it better to recall the definitions of stability of an equilibrium figure.

2.3. Free boundary problem in a layer.

\section{Initial boundary value problem for a layer}

Let be given the viscosity coefficient $\mu>0$ and the surface tension coefficient $\sigma>0$. The intensity of gravity acceleration is $g_{0}>0$ and the vector weight is always downward directed; thus its $x_{3}$ component will be negative or positive according as $\Gamma_{B}$ is situated below or above the free surface $\Gamma_{t}$, respectively. Also the external pressure is always positive, $q_{e}>0$, and density is taken equal to $1, \rho=1$. Let us find a domain $\Omega_{t}$ :

$$
\Omega_{t}=\left\{x=\left(x^{\prime}, x_{3}\right): x^{\prime} \in \Sigma, f\left(x^{\prime}\right)<x_{3}<h+\eta\left(x^{\prime}, t\right)\right\},
$$

and a solution $\mathbf{u}(x, t), q(x, t), \eta\left(x^{\prime}, t\right)$ satisfying the system

$$
\begin{array}{ll}
\partial_{t} \mathbf{u}+(\mathbf{u} \cdot \nabla) \mathbf{u}-\nabla \cdot T(\mathbf{u}, q)= \pm g_{0} \nabla x_{3} & \text { in } \Omega_{t}, t>0, \\
\nabla \cdot \mathbf{u}=0 & \text { in } \Omega_{t}, t>0, \\
T(\mathbf{u}, q) \mathbf{n}=\sigma \mathcal{H}(\eta) \mathbf{n}-q_{e} \mathbf{n} & \text { on } \Gamma_{t}, t>0, \\
\partial_{t} \eta=\mathbf{u} \cdot \tilde{\mathbf{n}} & \text { on } \Gamma_{t}, t>0, \\
\mathbf{u}=\mathbf{0} & \text { on } \Gamma_{B}, t>0, \\
\left.\mathbf{u}\right|_{t=0}=\mathbf{u}_{0} \text { in } \Omega_{0},\left.\quad \eta\right|_{t=0}=\eta_{0} \text { on } \Sigma . &
\end{array}
$$


Here $\mathbf{n}, \tilde{\mathbf{n}}$ are outer normals to $\Gamma_{t}$ defined by

$$
\mathbf{n}=\frac{1}{\sqrt{1+\left|\nabla^{\prime} \eta\right|^{2}}}\left(-\nabla^{\prime} \eta, 1\right), \quad \tilde{\mathbf{n}}=\sqrt{1+\left|\nabla^{\prime} \eta\right|^{2}} \mathbf{n}=\left(-\nabla^{\prime} \eta, 1\right)
$$

$\mathcal{H}$ is the doubled mean curvature of $\Gamma_{t}$ which is given by the formula

$$
\mathcal{H}(h+\eta)=\mathcal{H}(\eta)=\nabla^{\prime} \cdot\left(\frac{\nabla^{\prime} \eta}{\sqrt{1+\left|\nabla^{\prime} \eta\right|^{2}}}\right),
$$

where $\nabla^{\prime}$ is the gradient with respect to $x^{\prime}$. By the divergence free condition, the total mass is conserved:

$$
\frac{d}{d t}\left|\Omega_{t}\right|=\frac{d}{d t} \int_{\Omega_{t}} d x=\int_{\Gamma_{t}} \mathbf{n} \cdot \mathbf{u} d S=\int_{\Omega_{t}} \nabla \cdot \mathbf{u} d x=0 .
$$

Let the total volume filled by the liquid be given; thus the relation

$$
\left|\Omega_{b}\right|=\left|\Omega_{0}\right|=\int_{\Sigma} \int_{f\left(x^{\prime}\right)}^{h} d x_{3} d x^{\prime}=\int_{\Sigma}\left[h-f\left(x^{\prime}\right)\right] d x^{\prime}
$$

implies that $h$ is given 10 Moreover the condition that total volume is constant delivers

$$
\int_{\Sigma} \eta d x^{\prime}=\int_{\Sigma}\left[(h+\eta)-f\left(x^{\prime}\right)\right] d x^{\prime}-\left|\Omega_{b}\right|=0 .
$$

2.4. The rest state $S_{b}$. We consider the stationary problem associated to (2.16):

$$
\begin{array}{ll}
(\mathbf{v} \cdot \nabla) \mathbf{v}-\nabla \cdot T(\mathbf{v}, q)= \pm g_{0} \nabla x_{3} & \text { in } \Omega, t>0, \\
\nabla \cdot \mathbf{v}=0 & \text { in } \Omega, \\
T(\mathbf{v}, q) \mathbf{n}=\sigma \mathcal{H}(\eta) \mathbf{n}-q_{e} \mathbf{n} & \text { on } \Gamma, \\
\mathbf{v} \cdot \tilde{\mathbf{n}}=0 & \text { on } \Gamma, \\
\mathbf{v}=\mathbf{0} & \text { on } \Gamma_{B} .
\end{array}
$$

We look for exact solutions in the form $\mathbf{v}_{b}=\mathbf{0}$ and $\eta_{b}=$ const. From (2.20), we obtain $\eta_{b}=0, \zeta_{b}=h$ and $h$ is given fixing the volume occupied by the liquid $\Omega_{b}=\Omega$. Substituting $\mathbf{v}=\mathbf{0}$ in the momentum equation (2.21) ${ }_{1}$, we deduce that the pressure must have the form $q=q_{b}\left(x_{3}\right)= \pm g_{0} x_{3}+C$. On the free surface $\Gamma_{t}, q$ satisfies the condition $(2.21)_{3}$, which implies that $q_{b}(h)= \pm g_{0} h+C=q_{e}$; therefore,

$$
q_{b}\left(x_{3}\right)= \pm g_{0}\left(x_{3}-h\right)+q_{e} .
$$

We conclude that the set of stationary solutions of (2.21) is not empty; at least there exists the rest state $S_{b}$ :

$$
S_{b}=\left\{\mathbf{v}_{b}=\mathbf{0}, \eta_{b}=0, q_{b}\left(x_{3}\right)= \pm g_{0}\left(x_{3}-h\right)+q_{e}\right\},
$$

in the layer $\Omega_{b}=\left\{\left(x^{\prime}, x_{3}\right): x^{\prime} \in \Sigma, f\left(x^{\prime}\right)<x_{3}<h\right\}$, with $h$ given because the volume is given.

\footnotetext{
"In this computation, the external pressure is computed as a function of $h$; cf. [7]. There is an alternative approach where the external pressure is given and the height is computed afterward; cf. 21.
} 
3. Some definitions related to stability. Let $S_{b}$ denote an equilibrium solution of a portion of heavy liquid filling a horizontal layer. As is customary, the triple $S(t)=$ $(\mathbf{u}, \pi, \eta)$ defined by $\mathbf{u}(x, t)=\mathbf{v}(x, t)-\mathbf{v}_{b}(x), \pi(x, t)=q(x, t)-q_{b}(x), \eta=\zeta-h$ is called the perturbation to $S_{b}$. We may reduce the study of stability of $S_{b}$ to the study of stability of the zero solution $S_{0}=\{\mathbf{u}=0, \quad \pi=$ const., $\quad \eta=0\}$. It is known that stability studies the control for all time $t>0$ of a suitable norm of perturbation $S(t)$.

Let $\left(\mathbf{u}(x, t), \eta\left(x^{\prime}, t\right)\right)$ be a pair of regular functions, and set $\mathbf{u}_{0}(x)=\mathbf{u}(x, 0), \eta_{0}\left(x^{\prime}\right)=$ $\eta\left(x^{\prime}, 0\right)$. Given the metric $d(\zeta, \zeta-b):=\|\eta(\cdot, t)\|_{y}:=\left|\Gamma_{t}\right|-\Sigma \mid$, we introduce some norms:

$$
\begin{aligned}
& \|(\mathbf{u}, \eta)\|_{2}(t):=\|\mathbf{u}(\cdot, t)\|_{W^{1,2}\left(\Omega_{t}\right)}+\|\eta(\cdot, t)\|_{W^{1,2}(\Sigma)} \\
& \|(\mathbf{u}, \eta)\|_{\infty}(t):=\|\mathbf{u}(\cdot, t)\|_{W^{1,2}\left(\Omega_{t}\right)}+\|\eta(\cdot, t)\|_{W^{1, \infty}(\Sigma)}, \\
& \|(\mathbf{u}, \eta)\|_{0}(t):=\|\mathbf{u}(\cdot, t)\|_{L^{2}\left(\Omega_{t}\right)}+\|\eta(\cdot, t)\|_{y} \\
& \|(\mathbf{u}, \eta)\|_{1}(t):=\|\mathbf{u}(\cdot, t)\|_{W^{1,2}\left(\Omega_{t}\right)}+\|\eta(\cdot, t)\|_{y} \\
& \|(\mathbf{u}, \eta)\|_{X}:=\|(\mathbf{u}, \eta)\|_{i}, \quad \text { where } \quad i=1,2, \infty
\end{aligned}
$$

and we set

$$
\left\|\left(\mathbf{u}_{0}, \eta_{0}\right)\right\|_{X}:=\|(\mathbf{u}, \eta)\|_{X}(0) .
$$

With these norms we may define the set of solutions $(\mathbf{u}, \zeta)$ to (2.17) in the neighborhoods of the zero $\mathcal{I}_{r}^{i} \subseteq W^{1,2}\left(\Omega_{t}\right) \times W^{1, i}(\Sigma), i=1,2, \infty$, as the set of functions such that $\|(\mathbf{u}, \eta)\|_{X}(t)<r$.

Here we give some basic definitions concerning the control in time of $S(t)$ by means of initial data; such control is said to be stable with respect to the initial data.

Definition 3.1. The rest state $S_{b}$ is said to be nonlinearly stable in the norm $\|(\mathbf{u}, \eta)\|_{X}$ iff for all $\epsilon>0$ there exists a $\delta>0$ such that for all initial data $\left(\mathbf{u}_{0}, \eta_{0}\right)$ satisfying $\left\|\left(\mathbf{u}_{0}, \eta_{0}\right)\right\|_{X}<\delta$, we have

$$
\|(\mathbf{u}, \eta)\|_{X}(t)<\epsilon, \quad \forall t>0 .
$$

REMARK 3.1. It is easy to verify that $\mathcal{E}^{1 / 2}$ is equivalent to $\|(\mathbf{u}, \eta)\|_{1}$. However for regular motions, $\eta \in W^{1, \infty}(\Sigma), \mathcal{E}^{1 / 2}$ becomes equivalent to $\|(\mathbf{u}, \eta)\|_{2}$.

Definition 3.2. Let $\mathcal{E}^{1 / 2}(\mathbf{u}, \eta)$ be equivalent to the norm $\|(\mathbf{u}, \eta)\|_{2}(t)$; thus we call $\mathcal{E}^{1 / 2}(\mathbf{u}, \eta)$ the energy norm of perturbation. The rest state $S_{b}$ is said to be nonlinearly exponentially stable in the energy norm $\mathcal{E}^{1 / 2}(\mathbf{u}, \eta)$ iff there exists a $\delta>0$ such that for all initial data $\left(\mathbf{u}_{0}, \eta_{0}\right)$ satisfying $\mathcal{E}\left(\mathbf{u}_{0}, \eta_{0}\right)<\delta$ there exists a positive constant $b$ such that

$$
\mathcal{E}(\mathbf{u}, \eta)(t)<\mathcal{E}\left(\mathbf{u}_{0}, \eta_{0}\right) \exp ^{-b t}, \quad \forall t>0,
$$

$b$ is referred to as the decay constant.

DEFinition 3.3. The rest state is said to be nonlinearly unstable if it is not nonlinearly stable.

Let $\mathcal{E}_{L}(\mathbf{u}, \eta)(t)$ be the quadratic part of $\mathcal{E}(\mathbf{u}, \eta)(t)$; for the linearized problem associated to (2.17), see (7.1), all definitions can be repeated.

DEFinition 3.4. The rest state $S_{b}$ is said to be linearly stable in the norm $\mathcal{E}_{L}^{1 / 2}(\mathbf{u}, \eta)(t)$ iff there exists a constant $b>0$ such that

$$
\mathcal{E}_{L}(\mathbf{u}, \eta)(t)<c \mathcal{E}\left(\mathbf{u}_{0}, \eta_{0}\right) \exp ^{-b t}, \quad \forall t>0 .
$$


Definition 3.5. The rest state $S_{b}$ is said to be linearly unstable in the norm $\mathcal{E}_{L}^{1 / 2}(\mathbf{u}, \eta)(t)$ iff it is not linearly stable in the norm $\mathcal{E}_{L}^{1 / 2}(\mathbf{u}, \eta)(t)$.

We mention here two essential differences between linear and nonlinear stability with respect to the initial data in classical fluid dynamics.

The first is due to the size of the distance between the initial data $S(0)$ and $S_{b}$, which is the radius of a small ball in the study of nonlinear stability, while it has no bound in the study of linear stability.

The second is due to the decay rate of the energy norm of perturbations $\mathcal{E}(t)$, which is increased by $\mathcal{E}(0) f(t)$ with $f(t)$ bounded in the nonlinear case, and increased by $\mathcal{E}(0) \exp ^{-a t}$, with $a \geq 0, a=0$ for marginal stability, in the linear case.

Let us recall that by the "linearization principle" one means a theorem proving that for a nonlinear system, the stability properties of $S_{b}$ are deduced from its part linearized around $S_{b}$, provided the initial data are sufficiently close to $S_{b}$. Therefore, if a "linearization principle" holds for the rest state $S_{b}$, any linearly stable (unstable resp.) $S_{b}$ is also nonlinearly stable (unstable resp.), for a suitable class of small initial data.

If a linearization principle holds, then any linearly stable state $S_{b}$ is also nonlinearly stable. This means that solutions corresponding to initial data in a sufficiently small neighborhood of 0 remain close to 0 for all time. In reality we may prescribe large initial data; see our example in the introduction. Here we wish to study what happens also in this circumstance.

Here we make further remarks.

i) Notice that Definition 3.1 depends on the norm $\|(\mathbf{u}, \eta)\|_{X}(t)$, and on the radius $\delta$ of initial data; namely, it is a local statement.

ii) Notice that Definition 3.4 is independent of the size of the initial data; namely, it is a global statement.

iii) For regular solutions, linear and nonlinear stability do depend on the external forces.

iv) The difference between continuous dependence and stability lies on the time interval $(0, T)$.

Previous definitions on nonlinear stability say nothing about the control of solutions with finite initial data. Indeed in nonlinear phenomena, for large initial data, a solution $S(t)$ may lose its control from initial data, even though $S_{b}$ is nonlinearly stable (for small initial perturbations). This fact occurs frequently, and it constitutes the real discrepancy between linear and nonlinear stability. Up until today it appears that there are no rigorous definitions of this phenomena; thus we introduce here two new definitions:

Definition 3.6. A perturbation $(\mathbf{u}, \eta)$ to the rest state $S_{b}$ is said to be controlled by initial data in the range $\mathcal{I}_{2 a} / \mathcal{I}_{a}$ if and only if for all initial data $\left(\mathbf{u}_{0}, \eta_{0}\right)$ satisfying

$$
a<\left\|\left(\mathbf{u}_{0}, \eta_{0}\right)\right\|_{X}<2 a,
$$

the solution $\mathbf{u}, \eta$ corresponding to $\left(\mathbf{u}_{0}, \eta_{0}\right)$ is bounded for all time, namely, if there exists a suitable constant $\alpha=\alpha(a)>0$ such that

$$
\|(\mathbf{u}, \eta)\|_{X}(t) \leq \alpha, \quad \forall t>0 .
$$


DEFinition 3.7. The rest state is said to lose the control from the initial data iff there exists a large positive number $a$ and there exist initial data $\left(\mathbf{u}_{0}, \eta_{0}\right)$ satisfying (3.3), such that the corresponding solution $(\mathbf{u}(x, t), \eta(x, t))$ is not controlled by the initial data; namely, however, given $\alpha>0$, there exists $T>0$ such that the solution $(\mathbf{u}, \eta)$ of the problem (2.17) with initial data satisfying (3.3) satisfies the inequality

$$
\|(\mathbf{u}, \eta)\|_{X}(T) \geq \alpha
$$

REMARK 3.2. The definitions of control and loss of control from initial data include the definitions of nonlinear stability and instability.

I) Definitions 3.3 and 3.7 are meaningful only for nonlinear systems, because linear stability is global.

The aim of the present work is to relate the stability of the rest to the sign of the difference between the total energy of the perturbed flow at initial time and that of the rest state.

Here we wish to quote previous results about the stability of a fluid with a free surface. We notice that such results are available in the two cases of a layer and of a drop. Precisely, nonlinear stability results have been proved separately for a layer [20, [17, [18, and for a liquid drop [23, [24], [26]. A first nonlinear instability result under a technical assumption was proved in [19]. Several linear instability results for a liquid drop can be quoted [26]-28], [21], and the proof holds true also for a layer with rigid surface upward.

Let $\mathcal{V}$ be the following regularity class

$$
\begin{aligned}
\mathcal{V}= & \left\{\mathbf{u}(x, t), \eta\left(x^{\prime}, t\right), q(x, t):\right. \\
& \mathbf{u} \in L^{2}\left(0, \infty ; W_{\natural}^{1,2}\left(\Omega_{t}\right) \cap L^{\infty}\left(0, \infty ; L_{\natural}^{3}\left(\Omega_{t}\right)\right),\right. \\
& \left.\eta \in L^{\infty}\left(0, T ; W_{\natural}^{1, \infty}(\Sigma)\right),|\eta|<\frac{h}{4}, q \in L^{\infty}\left(0, T ; C_{\natural}^{0}\left(\bar{\Omega}_{t}\right)\right)\right\},
\end{aligned}
$$

where the symbol $\square$ denotes periodicity in the horizontal direction.

For a layer with a rigid surface below the liquid, Padula and Solonnikov have obtained the following theorem.

TheOrem 3.8 (Theorem 2.3 in [20]). Assume the fluid is above the horizontal layer and that there exist global solutions $(\mathbf{u}, \eta, q) \in \mathcal{V}$ to 2.16 corresponding to initial data $\left(\mathbf{u}_{0}, \eta_{0}\right)$. Then the rest state $S_{b}=\left\{\mathbf{u}_{b}=0, \eta_{b}=0, q_{b}\left(x_{3}\right)=-g_{0}\left(x_{3}-h\right)+\right.$ $\left.q_{e}\right\}$ is nonlinearly exponentially stable in the energy norm $\mathcal{E}^{1 / 2}(\mathbf{u}, \eta)$, in the class of perturbations belonging to $\mathcal{V}$. In this case the energy of perturbations is equivalent to a suitable norm of perturbations.

Notice that the hypotheses of Theorem 3.8 imply that the total energy is always positive.

From the results proved in 21] for a spherical geometry one can easily deduce in the simpler geometry of the layer the following linear instability result.

TheOREM 3.9 (From Theorem 1 in 21]). Let there exist a set of functions $\left(\mathbf{u}_{0}, \eta_{0}\right)$ such that the quadratic part $\mathcal{E}_{L}(\mathbf{u}, \eta)$ of the total energy assumes a negative value. 
Then the linearized form of problem (2.17) has solutions growing exponentially as $t \rightarrow \infty$. This means that the rest state $S_{b}$ is linearly unstable.

Now we observe that $\mathcal{E}_{L}(\mathbf{u}, \eta)$ is homogeneous of second grade; hence, for a given number $\lambda \in R$,

$$
\mathcal{E}_{L}(\lambda \mathbf{u}, \lambda \eta)=\lambda^{2} \mathcal{E}_{L}(\mathbf{u}, \eta)
$$

Thus, owing to (2.9) and (2.15) this yields

$$
\mathcal{E}(\lambda \mathbf{u}, \lambda \eta)=\lambda^{2} \mathcal{E}_{L}(\mathbf{u}, \eta)+o\left(\lambda^{2}\right),
$$

for $\lambda$ small enough, where the sign of $\mathcal{E}$ coincides with that of $\mathcal{E}_{L}$, and we have the following:

The hypotheses of Theorem 3.9 imply also that there exists a set of functions $\left(\lambda \mathbf{u}_{0}, \lambda \eta_{0}\right)$ such that the total energy is negative for small $\lambda$. A linearization principle is proved in section 5; in particular, if the rest state $S_{b}$ is linearly unstable, then it is also nonlinearly unstable. The assumptions of Theorem 3.9 are realized, e.g., by assuming that the fluid is below the horizontal layer and under suitable assumptions on ${ }^{* *} G=g_{0}|\Sigma| / \sigma$ and $\left|\Omega_{b}\right|=h|\Sigma|$.

Before giving our result we introduce the nondimensional characteristic number

$$
G:=\frac{g_{0}|\Sigma|}{\sigma}
$$

3.1. Present results. We begin by introducing the total energy of perturbation $\mathcal{E}(t)$

$$
\mathcal{E}(u(t), \eta(t)):=2 E_{U}(\eta(t))+\|u(\cdot, t)\|_{L^{2}\left(\Omega_{t}\right)}^{2},
$$

where $E_{U}$ has been defined in (2.4). In section 4 we prove an energy identity and notice that, if the initial energy is negative, it doesn't furnish an "a priori" estimate for the $L^{2}$-norms of solutions. In sections 5 and 6 we aim to prove the following results:

(i) In section 5, we prove two theorems: a nonlinear instability theorem; a loss of control on the initial data. Precisely, when there exists a set of initial data $\mathbf{u}_{0}$, $\eta_{0}$ such that the total energy $\mathcal{E}\left(\mathbf{u}_{0}, \eta_{0}\right)$ of perturbations at $t=0$ is negative, we prove the nonlinear instability Theorem 3.10 , without using a linearization principle. This is done in the wake of what was done in [21. Thus we introduce the definition of control of a solution through its initial data for all times $t>0$, and we prove Theorem 3.11 claiming that if there exist (may be large) initial data $\mathbf{u}_{0}, \eta_{0}$ such that $\mathcal{E}\left(\mathbf{u}_{0}, \eta_{0}\right)$ becomes negative, then the solution, corresponding to data $\mathbf{u}_{0}, \eta_{0}$, is not controlled by initial data.

(ii) In section 6 , we exhibit data $\sigma, g_{0} h$, such that: a) for all infinitesimal initial data $\left(\mathbf{u}_{0}, \eta_{0}\right)$, the total energy $\mathcal{E}_{0}=\mathcal{E}\left(\mathbf{u}_{0}, \eta_{0}\right)$ is positive; thus by Theorem 3.10, solutions corresponding to such $\mathcal{E}_{0}$ are nonlinearly stable; b) it is possible to construct initial data $\left(\mathbf{u}_{0}, \eta_{0}\right)$ larger than a computable constant such that the total energy $\mathcal{E}_{0}=\mathcal{E}\left(\mathbf{u}_{0}, \eta_{0}\right)$ is negative. Thus recalling Theorem 3.11 of section 4 , we claim that solutions corresponding to such initial data $\mathbf{u}_{0}, \eta_{0}$ are not controlled by initial data.

\footnotetext{
${ }^{* *}$ Notice that $G$ is a nondimensional number; cf. (2.6).
} 


\section{Result I.}

Theorem 3.10. Assume that for all $\delta>0$, there exist initial data $\left(\mathbf{u}_{0}, \eta_{0}\right)$ with $\left\|\left(\mathbf{u}_{0}, \eta_{0}\right)\right\|<\delta$, such that the initial energy $\mathcal{E}_{0}$ is negative,

$$
-\mathcal{E}_{0}=g_{0}\left\|\eta_{0}\right\|_{L^{2}(\Sigma)}^{2}-2 \sigma \int_{\Sigma}\left(\sqrt{1+\left|\nabla^{\prime} \eta_{0}\right|^{2}}-1\right) d x^{\prime}-\left\|\mathbf{u}_{0}\right\|_{L^{2}\left(\Omega_{0}\right)}^{2}>0 .
$$

Therefore there is some $\epsilon>0$ such that for all $\delta>0$ there exists an initial value $\left(\mathbf{u}_{0}, \eta_{0}\right) \in W^{1,2}\left(\Omega_{0}\right) \times W^{1, \infty}(\Sigma)$ less than $\delta$, and there exists $T>0$ such that the solution $(\mathbf{u}, \eta)$ of the problem (2.17) satisfies the inequality

$$
\|(\mathbf{u}, \eta)\|_{2}(T) \geq \epsilon .
$$

REMARK 3.3. Notice that, since the total energy of perturbation is a nonlinear functional of $(\mathbf{u}, \eta), \mathcal{E}_{0}$ may be negative for fixed functions $\left(\mathbf{u}_{*}, \eta_{*}\right)$, while its quadratic part may still be positive definite computed for the same functions $\left(\mathbf{u}_{*}, \eta_{*}\right)$.

REMARK 3.4. Notice that (3.6) is equivalent to the condition $G>G_{d}=\lambda_{1}=$ $4 \pi^{2} \min \left\{1,1 / d^{2}\right\}$, only for small initial data. We leave this proof to the interested reader.

We observe that the solution $(\mathbf{u}, \eta)$ to problem (2.17) is a perturbation to the rest.

Theorem 3.11. Given a constant $a$, let there exist $\left(\mathbf{u}_{0}, \eta_{0}\right)$ with $a<\left|\left(\mathbf{u}_{0}, \eta_{0}\right)\right|_{Y}<2 a$, which allows the initial energy $\mathcal{E}_{0}$ to be negative,

$$
-\mathcal{E}_{0}=g_{0}\left\|\eta_{0}\right\|_{L^{2}(\Sigma)}^{2}-2 \sigma \int_{\Sigma}\left(\sqrt{1+\left|\nabla^{\prime} \eta_{0}\right|^{2}}-1\right) d x^{\prime}-\left\|\mathbf{u}_{0}\right\|_{L^{2}\left(\Omega_{0}\right)}^{2}>0 .
$$

Then the solution $(\mathbf{u}, \eta)$ to problem (2.17) with initial data (3.3) is not controlled by the data.

\section{Result II.}

We construct a solution $(\mathbf{u}, \eta)$ such that, though linearly stable, it is not controlled by initial data when these data are larger than a computable constant $A$.

Theorem 3.12. Assume that the linear stability hypothesis holds:

$$
G<G_{d}:=\lambda_{1},
$$

where $\lambda_{1}$ is defined in subsection 6.1. We construct the initial values $\left(\mathbf{u}_{0}, \eta_{0}\right) \in W^{1,2}\left(\Omega_{0}\right) \times$ $W^{1, \infty}(\Sigma)$ satisfying the condition (3.8) ensuring that the initial energy $\mathcal{E}_{0}$ is negative.

In the wake of Theorem 3.11 we may claim:

There exists a positive large number a, such that the solution $(\mathbf{u}, \eta)$, corresponding to initial data $\left(\mathbf{u}_{0}, \eta_{0}\right)$ satisfying (3.3), is not controlled by the initial data, and for any $\alpha>0$ there exist initial data $\left(\mathbf{u}_{0}^{\alpha}, \eta_{0}^{\alpha}\right)$ satisfying (3.3), and an instant $T^{\alpha}>0$ such that the corresponding solution $\left(\mathbf{u}^{\alpha}, \eta^{\alpha}\right)$ to problem (2.17) satisfies the inequality

$$
\left\|\left(\mathbf{u}^{\alpha}, \eta^{\alpha}\right)\right\|_{X}\left(T^{\alpha}\right) \geq \alpha .
$$

In this paper we denote a generic constant by $C$ or $c$. They may change from line to line. 
4. Energy identity. We introduce the following regularity class:

$$
\begin{aligned}
\mathcal{W}= & \left\{\mathbf{u}(x, t), \eta\left(x^{\prime}, t\right), q(x, t):\right. \\
& \mathbf{u} \in L^{2}\left(0, \infty ; W_{\natural}^{1,2}\left(\Omega_{t}\right)\right) \cap L^{\infty}\left(0, \infty ; L_{\natural}^{3}\left(\Omega_{t}\right)\right), \\
& \left.\eta \in L^{\infty}\left(0, \infty ; W_{\natural}^{1, \infty}(\Sigma)\right), q \in L^{\infty}\left(0, \infty ; W_{\natural}^{1,2}\left(\Omega_{t}\right)\right)\right\} .
\end{aligned}
$$

Theorem 4.1. Let $(\mathbf{u}, \eta, q) \in \mathcal{W}$ satisfy (2.17). Then the energy identity

$$
\frac{d}{d t}\left[g_{0}|\Sigma|\|\eta\|_{L^{2}(\Sigma)}^{2}-2 \sigma \int_{\Sigma}\left(\sqrt{1+\left|\nabla^{\prime} \eta\right|^{2}}-1\right) d x^{\prime}-\|\mathbf{u}\|_{L^{2}\left(\Omega_{t}\right)}^{2}\right]=\mu\|S(\mathbf{u})\|_{L^{2}\left(\Omega_{t}\right)}^{2}
$$

or equivalently

$$
\frac{d}{d t}\left[g_{0}|\Sigma|\|\eta\|_{L^{2}(\Sigma)}^{2}-\sigma\left\|\nabla^{\prime} \eta\right\|_{L^{2}(\Sigma)}^{2}+\sigma\left(\mathcal{H}_{n l} \nabla^{\prime} \eta, \nabla^{\prime} \eta\right)_{\Sigma}-\|\mathbf{u}\|_{L^{2}\left(\Omega_{t}\right)}^{2}\right]=\mu\|S(\mathbf{u})\|_{L^{2}\left(\Omega_{t}\right)}^{2}
$$

holds, where $\mathcal{H}_{n l}$ is the nonlinear part of $\mathcal{H}$ which is given by

$$
\left(\mathcal{H}_{n l} \nabla^{\prime} \eta, \nabla^{\prime} \eta\right)_{\Sigma}=\int_{\Sigma} \frac{\sqrt{1+\left|\nabla^{\prime} \eta\right|^{2}}-1}{\sqrt{1+\left|\nabla^{\prime} \eta\right|^{2}}+1}\left|\nabla^{\prime} \eta\right|^{2} d x^{\prime} .
$$

REMARK 4.1. It is not difficult to check that the nonlinear part of $\mathcal{H}$ is positive, hence has a destabilizing effect; cf. 6.12).

Proof. To form the energy, we multiply (2.17) 1 by $\mathbf{u}$ and integrate over $\Omega_{t}$, which leads to

$$
\frac{1}{2} \frac{d}{d t} \int_{\Omega_{t}}|\mathbf{u}|^{2} d x=(\nabla \cdot T(\mathbf{u}, q), \mathbf{u})_{\Omega_{t}}+\left(g_{0} \nabla x_{3}, \mathbf{u}\right)_{\Omega_{t}},
$$

where $(\cdot, \cdot)_{\Omega_{t}}$ means the scalar product in $L^{2}\left(\Omega_{t}\right)$; see also (2.15). The left-hand side of (4.4) is obtained from the Reynolds transport theorem,

$$
\left(\partial_{t} \mathbf{u}, \mathbf{u}\right)_{\Omega_{t}}+(\mathbf{u} \cdot \nabla \mathbf{u}, \mathbf{u})_{\Omega_{t}}=\frac{1}{2} \frac{d}{d t} \int_{\Omega_{t}}|\mathbf{u}|^{2} d x
$$

Since the nonlinear term in (4.5) is estimated as

$$
\begin{aligned}
|(\mathbf{u} \cdot \nabla \mathbf{u}, \mathbf{u})|_{\Omega_{t}} & \leq \int_{\Omega_{t}}|\mathbf{u}|^{2}|\nabla \mathbf{u}| d x \leq\left(\int_{\Omega_{t}}|\mathbf{u}|^{4} d x\right)^{\frac{1}{2}}\left(\int_{\Omega_{t}}|\nabla \mathbf{u}|^{2} d x\right)^{\frac{1}{2}} \\
& =\|\mathbf{u}\|_{L^{4}\left(\Omega_{t}\right)}^{2}\|\nabla \mathbf{u}\|_{L^{2}\left(\Omega_{t}\right)} \leq C\|\mathbf{u}\|_{L^{3}\left(\Omega_{t}\right)}\|\nabla \mathbf{u}\|_{L^{2}\left(\Omega_{t}\right)}^{2},
\end{aligned}
$$

where we use the Sobolev inequality

$$
\|\mathbf{u}\|_{L^{4}\left(\Omega_{t}\right)}^{2} \leq C\|\mathbf{u}\|_{L^{3}\left(\Omega_{t}\right)}\|\nabla \mathbf{u}\|_{L^{2}\left(\Omega_{t}\right)}
$$

the calculations in (4.5) are meaningful. From the boundary condition (2.17) 3 ,

$$
\begin{aligned}
(\nabla \cdot T(\mathbf{u}, q), \mathbf{u})_{\Omega_{t}} & =(T(\mathbf{u}, q) \mathbf{n}, \mathbf{u})_{\Gamma_{t}}-\mu(S(\mathbf{u}), \nabla \mathbf{u})_{\Omega_{t}} \\
& =\left(\left(\sigma \mathcal{H}(\eta)-q_{e}\right) \mathbf{n}, \mathbf{u}\right)_{\Gamma_{t}}-\frac{\mu}{2}(S(\mathbf{u}), S(\mathbf{u}))_{\Omega_{t}} .
\end{aligned}
$$

Since

$$
\int_{\Sigma} \partial_{t} \eta d x^{\prime}=\int_{\Gamma_{t}} \partial_{t} \eta \frac{1}{\sqrt{1+\left|\nabla^{\prime} \eta\right|^{2}}} d S=\int_{\Gamma_{t}} \mathbf{n} \cdot \mathbf{u} d S=\int_{\Omega_{t}} \nabla \cdot u d x=0
$$


we have

$$
\begin{aligned}
& \int_{\Gamma_{t}}\left(\sigma \mathcal{H}(\eta)-q_{e}\right) \mathbf{n} \cdot \mathbf{u} d S=\int_{\Sigma}\left(\sigma \mathcal{H}(\eta)-q_{e}\right) \partial_{t} \eta d x^{\prime} \\
= & \int_{\Sigma} \sigma \mathcal{H}(\eta) \partial_{t} \eta d x^{\prime}=-\sigma \int_{\Sigma} \frac{\nabla^{\prime} \eta}{\sqrt{1+\left|\nabla^{\prime} \eta\right|^{2}}} \nabla^{\prime} \partial_{t} \eta d x^{\prime} \\
= & -\sigma \int_{\Sigma} \partial_{t} \frac{\left|\nabla^{\prime} \eta\right|^{2}}{2} \frac{1}{\sqrt{1+\left|\nabla^{\prime} \eta\right|^{2}}} d x^{\prime}=-\sigma \frac{d}{d t} \int_{\Sigma} \sqrt{1+\left|\nabla^{\prime} \eta\right|^{2}} d x^{\prime} .
\end{aligned}
$$

Moreover we obtain

$$
\begin{aligned}
\left(g_{0}|\Sigma| \nabla x_{3}, \mathbf{u}\right)_{\Omega_{t}} & =\left(g_{0}|\Sigma| \mathbf{n}(h+\eta), \mathbf{u}\right)_{\Gamma_{t}}-\left(g_{0}|\Sigma| x_{3}, \nabla \cdot \mathbf{u}\right)_{\Omega_{t}} \\
& =g_{0}|\Sigma| \int_{\Gamma_{t}}(h+\eta) \mathbf{n} \cdot \mathbf{u} d S=g_{0}|\Sigma| \int_{\Sigma}(h+\eta) \partial_{t} \eta d x^{\prime} \\
& =g_{0}|\Sigma| \frac{d}{d t} \int_{\Sigma} \frac{1}{2}(h+\eta)^{2} d x^{\prime}=g_{0}|\Sigma| \frac{d}{d t} \int_{\Sigma} \frac{1}{2} \eta^{2} d x^{\prime}
\end{aligned}
$$

Combining (4.4)-(4.10), we have

$$
\frac{d}{d t}\left[\frac{1}{2} \int_{\Omega_{t}}|\mathbf{u}|^{2} d x-\frac{g_{0}}{2} \int_{\Sigma} \eta^{2} d x^{\prime}+\sigma \int_{\Sigma} \sqrt{1+\left|\nabla^{\prime} \eta\right|^{2}} d x^{\prime}\right]+\frac{\mu}{2}\|S(\mathbf{u})\|_{L^{2}\left(\Omega_{t}\right)}^{2}=0,
$$

which is equivalent to (4.1).

We set

$$
D_{\eta}=\int_{\Sigma}\left(\sqrt{1+\left|\nabla \eta^{\prime}\right|^{2}}-1\right) d x^{\prime}
$$

Substituting (4.12) into (4.11) and changing sign, we get

$$
\frac{d}{d t}\left[g_{0}|\Sigma| \int_{\Sigma} \eta^{2} d x^{\prime}-2 \sigma D_{\eta}-\int_{\Omega_{t}}|\mathbf{u}|^{2} d x\right]=\mu\|S(\mathbf{u})\|_{L^{2}\left(\Omega_{t}\right)}^{2} .
$$

Since

$$
\frac{d}{d t} \int_{\Sigma} \sqrt{1+\left|\nabla^{\prime} \eta\right|^{2}} d x^{\prime}=\frac{d}{d t} \int_{\Sigma}\left(\sqrt{1+\left|\nabla^{\prime} \eta\right|^{2}}-1\right) d x^{\prime}=\frac{d}{d t} \int_{\Sigma} \frac{\left|\nabla^{\prime} \eta\right|^{2}}{\sqrt{1+\left|\nabla^{\prime} \eta\right|^{2}}+1} d x^{\prime}
$$

we obtain the dissipative effect of the nonquadratic part of $D_{\eta}$, namely

$$
\begin{aligned}
D_{\eta}=\int_{\Sigma} \frac{\left|\nabla^{\prime} \eta\right|^{2}}{\sqrt{1+\left|\nabla^{\prime} \eta\right|^{2}}+1} d x^{\prime} & =\int_{\Sigma}\left\{\frac{1}{2}+\left(\frac{1}{\sqrt{1+\left|\nabla^{\prime} \eta\right|^{2}}+1}-\frac{1}{2}\right)\right\}\left|\nabla^{\prime} \eta\right|^{2} d x^{\prime} \\
& =\frac{1}{2}\left\|\nabla^{\prime} \eta\right\|_{L^{2}(\Sigma)}^{2}-\frac{1}{2} \int_{\Sigma} \frac{\sqrt{1+\left|\nabla^{\prime} \eta\right|^{2}}-1}{\sqrt{1+\left|\nabla^{\prime} \eta\right|^{2}}+1}\left|\nabla^{\prime} \eta\right|^{2} d x^{\prime} \\
& =\frac{1}{2}\left\|\nabla^{\prime} \eta\right\|_{L^{2}(\Sigma)}^{2}-\frac{1}{2}\left(\mathcal{H}_{n l} \nabla^{\prime} \eta, \nabla^{\prime} \eta\right)_{\Sigma} .
\end{aligned}
$$

Thus by (4.11)-4.15) we obtain (4.2), which completes the proof of the theorem.

5. Proof of nonlinear instability for negative energy. In this section we prove Theorems 3.10 and 3.11 by absurdum. 
5.1. Proof of nonlinear instability for small initial data and negative energy. We assume that for every $\epsilon>0$ there exists a $\delta>0$ such that for all initial data with norm in $W^{1,2}\left(\Omega_{t}\right) \times W^{1, \infty}(\Sigma)$ less than $\delta$ the corresponding solution of (2.17) defined for all $t>0$ satisfies

$$
\|(\mathbf{u}, \eta)\|_{\infty}(t)=\|\mathbf{u}(\cdot, t)\|_{W^{1,2}\left(\Omega_{t}\right)}^{2}+\|\eta(\cdot, t)\|_{W^{1, \infty}(\Sigma)}^{2}<\epsilon, \quad \forall t>0 .
$$

Set

$$
K_{\eta}(t)=g_{0}|\Sigma|\|\eta\|_{L^{2}(\Sigma)}^{2}(t)-2 \sigma D_{\eta}(t) .
$$

Integrating (4.13) in $(0, t)$ yields

$$
K_{\eta}(t)=g_{0}|\Sigma|\|\eta\|_{L^{2}(\Sigma)}^{2}(t)-2 \sigma D_{\eta}(t)=\|\mathbf{u}\|_{L^{2}\left(\Omega_{t}\right)}^{2}(t)+\mu \int_{0}^{t}\|S(\mathbf{u})\|_{L^{2}(\Sigma)}^{2} d \tau+K_{0},
$$

where $K_{0}$ is given by (3.6):

$$
K_{0}=g_{0}|\Sigma|\left\|\eta_{0}\right\|_{L^{2}(\Sigma)}^{2}-2 \sigma \int_{\Sigma}\left(\sqrt{1+\left|\nabla^{\prime} \eta_{0}\right|^{2}}-1\right) d x^{\prime}-\left\|\mathbf{u}_{0}\right\|_{L^{2}\left(\Omega_{0}\right)}^{2},
$$

which is positive by assumption. We take $\mathbf{u}_{0}=0$; thus $K_{\eta}(0)=K_{0}$. Since $\eta$ satisfies the average zero condition (2.20), the generalized Poincaré inequality

$$
\|\eta\|_{L^{2}(\Sigma)}^{2} \leq C_{d}\left\|\nabla^{\prime} \eta\right\|_{L^{2}(\Sigma)}^{2}
$$

holds (cf. (2.1)). Furthermore we obtain the inequality

$$
\sqrt{1+p^{2}} \leq 1+|p|
$$

From the identity

$$
D_{\eta}=\int_{\Sigma} \frac{\left[\sqrt{1+\left|\nabla^{\prime} \eta\right|^{2}}-1\right]\left[\sqrt{1+\left|\nabla^{\prime} \eta\right|^{2}}+1\right]}{1+\sqrt{1+\left|\nabla^{\prime} \eta\right|^{2}}} d x^{\prime}=\int_{\Sigma} \frac{\left|\nabla^{\prime} \eta\right|^{2}}{1+\sqrt{1+\left|\nabla^{\prime} \eta\right|^{2}}} d x^{\prime},
$$

(5.4), and the hypotheses (5.1), (5.5) with $p=\left|\nabla^{\prime} \eta\right|<\epsilon$ we obtain, for $C_{d}=G_{d}^{-1}$, with $G_{d}$ defined in (6.8),

$$
\|\eta\|_{L^{2}(\Sigma)}^{2} \leq C_{d}\left\|\nabla^{\prime} \eta\right\|_{L^{2}(\Sigma)}^{2}=C_{d} \int_{\Sigma} \frac{\left|\nabla^{\prime} \eta\right|^{2}}{1+\sqrt{1+\left|\nabla^{\prime} \eta\right|^{2}}}\left[1+\sqrt{1+\left|\nabla^{\prime} \eta\right|^{2}}\right] d x^{\prime} \leq C_{d}(2+\epsilon) D_{\eta} .
$$

Furthermore from (5.3) we have

$$
D_{\eta}(t)<\frac{g_{0}|\Sigma|}{2 \sigma}\|\eta\|_{L^{2}(\Sigma)}^{2}(t)
$$

Inequalities (5.7) and (5.8) imply

$$
\frac{1}{C_{d}(2+\epsilon)}\|\eta\|_{L^{2}(\Sigma)}^{2}(t) \leq D_{\eta}(t)<\frac{g_{0}|\Sigma|}{2 \sigma}\|\eta\|_{L^{2}(\Sigma)}^{2}(t),
$$

namely (5.7), (5.8) imply the equivalence between the two norms of $\eta,\|\nabla \eta\|_{y}$, and $\|\nabla \eta\|_{L^{2}(\Sigma)}$. Therefore we claim that for any $t$ there exists a number $l(t)$ such that

$$
l(t)\|\eta\|_{L^{2}(\Sigma)}^{2}(t)=D_{\eta}(t), \quad \frac{1}{C_{d}(2+\epsilon)} \leq l(t)<\frac{g_{0}|\Sigma|}{2 \sigma} .
$$


We note that $g_{0}>G_{d} \sigma$ from the assumption (3.6) of Theorem 3.10. Combining (5.2) and (5.10), we obtain

$$
K_{\eta}(t)=\left(g_{0}-2 \sigma l(t)\right)\|\eta\|_{L^{2}(\Sigma)}^{2}(t) .
$$

Substituting (5.11) into (5.3), and using (5.1), we obtain

$$
g_{0}-2 \sigma l(t)=\frac{K_{\eta}(t)}{\|\eta\|_{L^{2}(\Sigma)}^{2}(t)}>\frac{K_{0}}{\epsilon} .
$$

From (5.11), (5.12) it follows that

$$
K_{\eta}(t)>\frac{K_{0}}{\epsilon}\|\eta\|_{L^{2}(\Sigma)}^{2}(t) .
$$

Now we use the free work equation. Let us multiply (2.17), by $\mathbf{V}$, constructed in Lemma 7.3 , and integrate over $\Omega_{t}$. This gives

$$
\frac{d}{d t}(\mathbf{u}, \mathbf{V})_{\Omega_{t}}+\int_{\Gamma_{t}}\left(-\sigma \mathcal{H}(\eta)-g_{0}|\Sigma| \eta\right) \mathbf{V} \cdot \mathbf{n} d S=\mathcal{F}(\mathbf{u}, \mathbf{V})(t)
$$

where

$$
\mathcal{F}(\mathbf{u}, \mathbf{V})=\left(\mathbf{u}, \partial_{t} \mathbf{V}\right)_{\Omega_{t}}+((\mathbf{u} \cdot \nabla) \mathbf{V}, \mathbf{u})_{\Omega_{t}}-\mu(S(\mathbf{u}), \nabla \mathbf{V})_{\Omega_{t}} .
$$

Integrating by parts in the surface integral in (5.14), and recalling the boundary value of $\mathbf{V}$, this yields

$$
\frac{d}{d t}(\mathbf{u}, \mathbf{V})_{\Omega_{t}}=-\int_{\Sigma}\left(\frac{\sigma}{\sqrt{1+\left|\nabla^{\prime} \eta\right|^{2}}}\left|\nabla^{\prime} \eta\right|^{2}-g_{0}|\Sigma||\eta|^{2}\right) d x^{\prime}+\mathcal{F}(\mathbf{u}, \mathbf{V})(t) .
$$

It follows that

$$
\int_{\Sigma} \frac{\sigma}{\sqrt{1+\left|\nabla^{\prime} \eta\right|^{2}}}\left|\nabla^{\prime} \eta\right|^{2} d x^{\prime}<\int_{\Sigma} \frac{2 \sigma}{1+\sqrt{1+\left|\nabla^{\prime} \eta\right|^{2}}}\left|\nabla^{\prime} \eta\right|^{2} d x^{\prime}=2 \sigma D_{\eta}(t)
$$

and therefore

$$
\frac{d}{d t}(\mathbf{u}, \mathbf{V})_{\Omega_{t}}>g_{0}|\Sigma|\|\eta\|_{L^{2}(\Sigma)}^{2}(t)-2 \sigma D_{\eta}(t)+\mathcal{F}(\mathbf{u}, \mathbf{V})(t)=K_{\eta}(t)+\mathcal{F}(\mathbf{u}, \mathbf{V})(t)
$$

Let us multiply by an arbitrary positive number $\gamma$ (5.17) and add it to (4.13) to have

$$
\frac{d}{d t} z(t)>\mu\|S(\mathbf{u})\|_{L^{2}\left(\Omega_{t}\right)}^{2}(t)+\gamma K_{\eta}(t)+\gamma \mathcal{F}(\mathbf{u}, \mathbf{V})(t),
$$

where

$$
z(t)=K_{\eta}(t)-\|\mathbf{u}\|_{L^{2}\left(\Omega_{t}\right)}^{2}(t)+\gamma(\mathbf{u}, \mathbf{V})_{\Omega_{t}}(t) .
$$

Now we estimate $\mathcal{F}(\mathbf{u}, \mathbf{V})$. Since $\mathbf{u}=0$ on $\Gamma_{B}$, Poincaré's inequality

$$
\|\mathbf{u}\|_{L^{2}\left(\Omega_{t}\right)} \leq \sqrt{C_{d}}\|\nabla \mathbf{u}\|_{L^{2}\left(\Omega_{t}\right)}
$$

holds. By (5.20) and Korn's inequality, we obtain

$$
\|\mathbf{u}\|_{L^{2}\left(\Omega_{t}\right)} \leq C\|S(\mathbf{u})\|_{L^{2}\left(\Omega_{t}\right)} .
$$

By (2.17), (7.16), (15.20) and Korn's inequality, we obtain

$$
\begin{aligned}
\left\|\partial_{t} \mathbf{V}\right\|_{L^{2}\left(\Omega_{t}\right)} & \leq C\left\|\partial_{t} \eta\right\|_{L^{2}(\Sigma)} \leq C\|\mathbf{u} \cdot \mathbf{n}\|_{L^{2}\left(\Gamma_{t}\right)} \leq C\|\mathbf{u}\|_{W^{1,2}\left(\Omega_{t}\right)} \\
& \leq C\|\nabla \mathbf{u}\|_{L^{2}\left(\Omega_{t}\right)} \leq C\|S(\mathbf{u})\|_{L^{2}\left(\Omega_{t}\right)} .
\end{aligned}
$$


By Sobolev's embedding theorem it follows that

$$
W^{1,2}\left(\Omega_{t}\right) \subset L^{3}\left(\Omega_{t}\right) .
$$

Remark 5.1. Notice that in general the Poincaré and the Korn constants are functions of $\Omega_{t}$ and therefore of time. However, by hypothesis (5.1), these constants become less than a constant in time.

By using (4.6), $((\mathbf{u} \cdot \nabla) \mathbf{V}, \mathbf{u})_{\Omega_{t}}$ is estimated by

$$
\begin{aligned}
\left|((\mathbf{u} \cdot \nabla) \mathbf{V}, \mathbf{u})_{\Omega_{t}}\right| & \leq \int_{\Omega_{t}}|\mathbf{u}|^{2}|\nabla \mathbf{V}| d x \\
& \leq\left(\int_{\Omega_{t}}|\mathbf{u}|^{4} d x\right)^{\frac{1}{2}}\left(\int_{\Omega_{t}}|\nabla \mathbf{V}|^{2} d x\right)^{\frac{1}{2}}=\|\mathbf{u}\|_{L^{4}\left(\Omega_{t}\right)}^{2}\|\nabla \mathbf{V}\|_{L^{2}\left(\Omega_{t}\right)} \\
& \leq C_{1}\|\mathbf{u}\|_{L^{3}\left(\Omega_{t}\right)}\|\nabla \mathbf{u}\|_{L^{2}\left(\Omega_{t}\right)}\|\nabla \mathbf{V}\|_{L^{2}\left(\Omega_{t}\right)} .
\end{aligned}
$$

By (5.21), (5.22), (5.24), (7.17), (5.1), and (5.23), we obtain

$$
\begin{aligned}
&|\mathcal{F}(\mathbf{u}, \mathbf{V})| \leq\left|\left(\mathbf{u}, \partial_{t} \mathbf{V}\right)_{\Omega_{t}}+\right|((\mathbf{u} \cdot \nabla) \mathbf{V}, \mathbf{u})_{\Omega_{t}}|+\mu|(S(\mathbf{u}), \nabla \mathbf{V})_{\Omega_{t}} \mid \\
& \leq\|\mathbf{u}\|_{L^{2}\left(\Omega_{t}\right)}\left\|\partial_{t} \mathbf{V}\right\|_{L^{2}\left(\Omega_{t}\right)}+C_{1}\|\mathbf{u}\|_{L^{3}\left(\Omega_{t}\right)}\|\nabla \mathbf{u}\|_{L^{2}\left(\Omega_{t}\right)}\|\nabla \mathbf{V}\|_{L^{2}\left(\Omega_{t}\right)}+\mu\|S(\mathbf{u})\|_{L^{2}\left(\Omega_{t}\right)}\|\nabla \mathbf{V}\|_{L^{2}\left(\Omega_{t}\right)} \\
& \leq C\|S(\mathbf{u})\|_{L^{2}\left(\Omega_{t}\right)}^{2}+c C_{1}\|\mathbf{u}\|_{L^{3}\left(\Omega_{t}\right)}\|S(\mathbf{u})\|_{L^{2}\left(\Omega_{t}\right)}\left\|\nabla^{\prime} \eta\right\|_{L^{2}(\Sigma)} \\
& \quad+c \mu\|S(\mathbf{u})\|_{L^{2}\left(\Omega_{t}\right)}\left\|\nabla^{\prime} \eta\right\|_{L^{2}(\Sigma)} \\
& \leq C\|S(\mathbf{u})\|_{L^{2}\left(\Omega_{t}\right)}^{2}+c\left(C_{1} \epsilon+\mu\right)\|S(\mathbf{u})\|_{L^{2}\left(\Omega_{t}\right)}\left\|\nabla^{\prime} \eta\right\|_{L^{2}(\Sigma)} .
\end{aligned}
$$

We recall that, by (5.11) and (5.13),

$$
K_{\eta}(t)=\left(g_{0}-2 \sigma l(t)\right)\|\eta\|_{L^{2}(\Sigma)}^{2}(t)>\frac{K_{0}}{\epsilon}\|\eta\|_{L^{2}(\Sigma)}^{2}(t) .
$$

Combining (5.12), (5.8) and (5.26), it follows that

$$
\begin{aligned}
\left\|\nabla^{\prime} \eta\right\|_{L^{2}(\Sigma)}^{2}(t) & \leq(2+\epsilon) D_{\eta}(t)<(2+\epsilon) \frac{g_{0}|\Sigma|}{2 \sigma}\|\eta\|_{L^{2}(\Sigma)}^{2}(t) \\
& <(2+\epsilon) \frac{g_{0}|\Sigma|}{2 \sigma} \frac{\epsilon}{K_{0}} K_{\eta}(t) .
\end{aligned}
$$

Applying Cauchy's inequality to (5.25) and using (5.27), we get

$$
\begin{aligned}
\gamma \mathcal{F}(\mathbf{u}, \mathbf{V}) \leq|\gamma \mathcal{F}(\mathbf{u}, \mathbf{V})| & \leq\left(\gamma C+\frac{\mu}{2}\right)\|S(\mathbf{u})\|_{L^{2}\left(\Omega_{t}\right)}^{2}+\gamma^{2} c^{2} \frac{\left(C_{1} \epsilon+\mu\right)^{2}}{2 \mu}\left\|\nabla^{\prime} \eta\right\|_{L^{2}(\Sigma)}^{2} \\
& <\left(\gamma C+\frac{\mu}{2}\right)\|S(\mathbf{u})\|_{L^{2}\left(\Omega_{t}\right)}^{2}+\gamma^{2} c^{2} \frac{\left(C_{1} \epsilon+\mu\right)^{2}}{2 \mu}(2+\epsilon) \frac{g_{0}|\Sigma|}{2 \sigma} \frac{\epsilon}{K_{0}} K_{\eta}
\end{aligned}
$$

hence we deduce

$$
-\gamma \mathcal{F}(\mathbf{u}, \mathbf{V})(t) \geq-\left(\frac{\mu}{2}+\gamma C\right)\|S(\mathbf{u})\|_{L^{2}(\Sigma)}^{2}(t)-\gamma^{2} c^{2} \frac{\left(C_{1} \epsilon+\mu\right)^{2}}{2 \mu}(2+\epsilon) \frac{g_{0}|\Sigma|}{2 \sigma} \frac{\epsilon}{K_{0}} K_{\eta}(t)
$$


The free work inequality (5.18) implies that

$$
\frac{d}{d t} z(t)>\left(\frac{\mu}{2}-\gamma C\right)\|S(\mathbf{u})\|_{L^{2}(\Sigma)}^{2}(t)+\gamma\left(1-\gamma c^{2} \frac{\left(C_{1} \epsilon+\mu\right)^{2}}{2 \mu}(2+\epsilon) \frac{g_{0}}{2 \sigma} \frac{\epsilon}{K_{0}}\right) K_{\eta}(t) .
$$

If we choose $\gamma$ so small that $\gamma C<\mu / 2$, and

$$
1-\gamma c^{2} \frac{\left(C_{1} \epsilon+\mu\right)^{2}}{2 \mu}(2+\epsilon) \frac{g_{0}|\Sigma|}{2 \sigma} \frac{\epsilon}{K_{0}}>\frac{1}{2},
$$

then we get

$$
\frac{d}{d t} z(t)>\frac{\gamma}{2} K_{\eta}(t)
$$

By (5.19), Cauchy's inequality, (5.26) and (7.18), we obtain

$$
\begin{aligned}
z(t)=K_{\eta}(t)- & \|\mathbf{u}\|_{L^{2}\left(\Omega_{t}\right)}^{2}(t)+\gamma(\mathbf{u}, \mathbf{V})_{\Omega_{t}}(t) \\
& \leq K_{\eta}(t)+\frac{C \gamma^{2}}{4}\|\eta\|_{W^{1,2}(\Sigma)}^{2}(t)<\left(1+\frac{\gamma^{2} C \epsilon}{4 K_{0}}\right) K_{\eta}(t) .
\end{aligned}
$$

Combining (5.30) with (5.31), we obtain

$$
\frac{d}{d t} z(t)>\frac{\gamma}{2} \frac{1}{1+\frac{\gamma^{2} C \epsilon}{4 K_{0}}} z(t)
$$

Below we show that, by applying Gronwall's Lemma to (5.32), we obtain an absurdum. Actually we set

$$
\beta=\frac{\gamma}{2} \frac{1}{1+\frac{\gamma^{2} C \epsilon}{4 K_{0}}} .
$$

By (5.19), (5.11) and (7.18), it follows that

$$
\begin{aligned}
z(t) & =K_{\eta}(t)-\|\mathbf{u}\|_{L^{2}\left(\Omega_{t}\right)}^{2}(t)+\gamma(\mathbf{u}, \mathbf{V})_{\Omega_{t}}(t) \\
& \leq K_{\eta}(t)+\gamma(\mathbf{u}, \mathbf{V})_{\Omega_{t}}(t) \\
& \leq\left(g_{0}-2 \sigma l(t)\right)\|\eta\|_{L^{2}(\Sigma)}^{2}(t)+\frac{c \gamma}{2}\|\mathbf{u}\|_{L^{2}\left(\Omega_{t}\right)}^{2}(t)+\frac{\gamma}{2}\|\eta\|_{W^{1,2}(\Sigma)}^{2}(t) \\
& \leq\left(g_{0}+\frac{c \gamma}{2}\right)\left(\|\mathbf{u}\|_{L^{2}\left(\Omega_{t}\right)}^{2}+\|\eta\|_{W^{1,2}(\Sigma)}^{2}\right)(t) .
\end{aligned}
$$

We now choose initial data suitably, and fix $\mathbf{u}_{0}=0$, so that $z(0)=K_{\eta}(0)>$ $\left(K_{0} / \epsilon\right)\left\|\eta_{0}\right\|_{L^{2}(\Sigma)}^{2}$.

For any given $\epsilon>0$, there exists time $T>0$ such that

$$
\begin{aligned}
e^{\beta T}\left(g_{0}+\frac{c \gamma}{2}\right) C \epsilon<e^{\beta T} z(0) \leq z(T) & \leq\left(g_{0}+\frac{c \gamma}{2}\right)\left(\|\mathbf{u}\|_{L^{2}\left(\Omega_{T}\right)}^{2}+\|\eta\|_{W^{1,2}(\Sigma)}^{2}\right)(T) \\
& \leq\left(g_{0}+\frac{c \gamma}{2}\right)\left(\|\mathbf{u}\|_{W^{1,2}\left(\Omega_{T}\right)}^{2}+\|\eta\|_{W^{1,2}(\Sigma)}^{2}\right)(T) .
\end{aligned}
$$

This contradicts (5.1), which completes the proof of the theorem. 
5.2. Loss of control of solution for large initial data and negative energy. In this subsection we prove Theorem 3.11. Since the proof of instability reduces to computing the sign of the energy of perturbations, we may follow the previous proof. In the wake of the proof of Theorem 3.10 also here the proof is given by absurdum. We assume that a solution $(\mathbf{u}, \eta)$ of (2.17) defined for all $t>0$ has a norm uniformly bounded in time, i.e. that it satisfies

$$
\|\mathbf{u}\|_{W^{1,2}\left(\Omega_{t}\right)}^{2}(t)+\|\eta\|_{W^{1, \infty}(\Sigma)}^{2}(t)<2 \alpha, \quad t \in(0, \infty) .
$$

By carefully repeating all steps of the previous section 3 by interchanging $\epsilon$ with $\alpha$, it is not difficult to prove that for any given $\alpha>0$, there exists time $T>0$ such that

$$
\begin{aligned}
e^{\beta T}\left(g_{0}+\frac{\gamma}{2}\right) C \alpha<e^{\beta T} z(0) \leq z(T) & \leq\left(g_{0}+\frac{\gamma}{2}\right)\left(\|\mathbf{u}\|_{L^{2}\left(\Omega_{T}\right)}^{2}+\|\eta\|_{W^{1,2}(\Sigma)}^{2}\right)(T) \\
& \leq\left(g_{0}+\frac{\gamma}{2}\right)\left(\|\mathbf{u}\|_{W^{1,2}\left(\Omega_{T}\right)}^{2}+\|\eta\|_{W^{1,2}(\Sigma)}^{2}\right)(T) .
\end{aligned}
$$

This contradicts (5.34), which completes the proof of the theorem.

6. $\mathcal{E}(0, \tau)$ positive for small, negative for large perturbations to $\mathcal{F}_{*}$. In this part, we give an example of initial values and characteristic parameters $\sigma, g_{0}, d, h$ ensuring a positive linearized initial energy of perturbations $\mathcal{E}_{0}$; namely, we give an example of a rest state with characteristic parameters $\sigma, g_{0}, d, h$ ensuring linear stability. Next for the same set of characteristic parameters $\sigma, g_{0}, d, h$ we construct sufficiently large initial data ensuring that the total energy of perturbations at the initial time is negative. This latter result is reached provided the shape of the liquid drop is suitable; cf. 6.27) 1 In the sequel, we shall not change symbols and we work with the same equations.

In the sequel we use the notations

$$
\mathcal{E}(0, \tau)=\sigma E_{0}(\tau), \quad G_{d}:=4 \pi^{2} \min \left\{1, \frac{1}{d^{2}}\right\} .
$$

For Sobolev spaces $X$ a subscript $0, X_{0}$, represents the same space quotient with respect to constants. Here we consider a flat rigid section $\Sigma \times 0$, and we prove the following theorems.

Theorem 6.1. It is possible to determine suitable values of $g_{0}, \sigma, h, d$ such that:

(1)

$$
G<G_{d}
$$

(2) there exists a positive constant $\delta\left(g_{0}, \sigma, h, d\right)>0$ such that for all $\tau \in L^{\infty}((0, \infty)$, $\left.W^{1, \infty}(\Sigma)\right)$ satisfying

$$
\int_{\Sigma} \tau d x^{\prime}=0, \quad h+\tau>\frac{h}{2}, \quad\|\tau\|_{W^{1, \infty}(\Sigma)}<\delta
$$

\footnotetext{
${ }^{\dagger \dagger}$ We shall work with nondimensional quantities; therefore setting $\widetilde{x}_{1}:=x_{1} / m t, \widetilde{x}_{2}:=x_{2} / d m t$, where $m t$ means meter, it will be

$$
\partial_{x_{1}}=\frac{1}{m t} \partial_{\widetilde{x}_{1}}, \quad \partial_{x_{2}}=\frac{1}{d m t} \partial_{\widetilde{x}_{2}} .
$$
}


we verify also that

$$
E(\tau)>0
$$

Theorem 6.2. It is possible to determine suitable values of $g_{0}, \sigma, h, d$ such that:

(1)

$$
G<G_{d}
$$

(2) there exists a denumerable number of functions $\tau\left(g_{0}, \sigma, h, d\right)>0$, sufficiently large, with $\tau \in L^{\infty}\left((0, \infty), W^{1, \infty}(\Sigma)\right)$ satisfying

$$
\int_{\Sigma} \tau d x^{\prime}=0, \quad h+\tau>\frac{h}{2},
$$

such that the nonlinear total energy of perturbation $\mathcal{E}$ computed in $(0, \tau)$ is negative,

$$
E(\tau)<0 .
$$

6.1. Construction of a basis. We begin by observing that it is possible to find in $L_{0}^{2}(\Sigma)$ an orthonormal basis of functions $\tau_{k}$ and a sequence $\lambda_{k} \in R$ in such a way that it yields

$$
-\Delta^{\prime} \tau_{k}-G \tau_{k}=\lambda_{k} \tau_{k}, \quad \int_{\Sigma} \tau_{k} d x^{\prime}=0
$$

In particular, we choose $\tau_{k}$, as a product of periodic functions $f\left(2 i \pi x_{1}\right) g\left(\frac{2 j \pi x_{2}}{d}\right), f, g \in$ $\{\sin , \cos \}, i, j \in \mathbb{N}_{0}, k=k(i, j),(i, j) \neq(0,0)$, multiplied by coefficients $\alpha_{i j}$ that provide unitary norms. Also the smallest of the corresponding eigenvalues is given by

$$
\lambda_{1}=G_{d}-G,
$$

where

$$
G_{d}:=4 \pi^{2} \min \left\{1, \frac{1}{d^{2}}\right\} .
$$

Hence we have the following equivalent inequalities:

$$
G<G_{d}:=4 \pi^{2} \min \left\{1, \frac{1}{d^{2}}\right\} \quad \Longleftrightarrow \quad \lambda_{1}=G_{d}-G>0 .
$$

This yields $\lambda_{k} \geq \lambda_{1}>0, \forall k \in N$ In our proofs the hypothesis

$$
\lambda_{1}>0
$$

is essential; this explains the request that eigenfunctions must have zero mean values, say, when we work in the quotient space of $L^{2}(\Sigma)$.

$\ddagger \ddagger$ This result can be read to mean: that for a liquid layer below a rigid plane, (6.2) is certainly true under the linear stability (3.9) hypothesis:

$$
\frac{g_{0}|\Sigma|}{\sigma}<4 \pi^{2} \min \left\{1, \frac{1}{d^{2}}\right\} .
$$

Namely, the linearized total energy of perturbation is positive for every regular function. 
We notice that multiplying (6.6) times $\tau_{k}$, integrating over $\Sigma$, and integrating by parts, it furnishes

$$
\begin{aligned}
& \left(\lambda_{k}+G\right) \int_{\Sigma} \tau_{k}^{2} d x^{\prime}=-\int_{\Sigma} \tau_{k} \Delta^{\prime} \tau_{k} d x^{\prime} \\
& =\int_{\Sigma}\left|\nabla^{\prime} \tau_{k}\right|^{2} d x^{\prime}-\int_{\partial \Sigma} \tau_{k} \nabla^{\prime} \tau_{k} \cdot \nu d l=\int_{\Sigma}\left|\nabla^{\prime} \tau_{k}\right|^{2} d x^{\prime} .
\end{aligned}
$$

Notice that the line integral over $\partial \Sigma$ becomes zero when $\tau_{k}$ vanishes (Dirichlet condition) and when the normal derivative of $\tau_{k}$ is zero (Neumann condition) or, which is our case, when the functions are periodic.

Let $\mathbf{u}_{0}=0$. The total energy reduces to

$$
\begin{aligned}
& E\left(\eta_{0}\right)=\left(2 \int_{\Sigma}\left(\sqrt{1+\left|\nabla^{\prime} \eta_{0}\right|^{2}}-1\right) d x^{\prime}-G \int_{\Sigma} \eta_{0}^{2} d x^{\prime}\right), \\
& G=\frac{g_{0}|\Sigma|}{\sigma}<4 \pi^{2} \min \left\{1, \frac{1}{d^{2}}\right\}=G_{d} .
\end{aligned}
$$

Then the initial energy is given by $\mathcal{E}\left(0, \eta_{0}\right)=\sigma E\left(\eta_{0}\right)$. Of course the sign of $\left.E \eta_{0}\right)$ depends on the parameters $\sigma, g_{0}, h, d$. In the sequel we set $\eta_{0}=\tau$.

The aim of this computation is twofold:

a) Theorem 6.1 to prove that there exists $\varepsilon>0$ such that for all $\tau \in C^{1}(\Sigma)$ satisfying the conditions

$$
\left.\tau\right|_{\partial \Sigma}=0, \quad \int_{\Sigma} \tau d x^{\prime}=0, \quad\left\|\nabla^{\prime} \tau\right\|_{\infty}=\sup _{\Sigma}\left|\nabla^{\prime} \tau\right|<\varepsilon
$$

we have $E(\tau) \geq 0$

b) Theorem 6.2 to prove that there exists $\alpha>0$, and a $\tau$ such that if $\tau \in C^{1}(\Sigma)$ satisfies the conditions

$$
\left.\tau\right|_{\partial \Sigma}=0, \quad \int_{\Sigma} \tau d x^{\prime}=0, \quad\left\|\nabla^{\prime} \tau\right\|_{\infty}=\sup _{\Sigma}\left|\nabla^{\prime} \tau\right|>\alpha,
$$

then we have $E(\tau)<0$.

6.2. Proof of Theorem 6.1: Positiveness of $E\left(\eta_{0}\right)$ for small initial data. We notice that

$$
\begin{aligned}
\sqrt{1+p^{2}}-1 & =\frac{p^{2}}{\sqrt{1+p^{2}}+1}=p^{2}\left(\frac{1}{2}+\frac{1}{\sqrt{1+p^{2}}+1}-\frac{1}{2}\right) \\
& =\frac{p^{2}}{2}+p^{2} \frac{1-\sqrt{1+p^{2}}}{2\left(1+\sqrt{1+p^{2}}\right)}=\frac{p^{2}}{2}-\frac{p^{4}}{2\left(1+\sqrt{1+p^{2}}\right)^{2}}=\frac{p^{2}}{2}-R(p) .
\end{aligned}
$$

Since $1+\sqrt{1+p^{2}} \geq 2$, the following estimate is true:

$$
R(p)=|R(p)| \leq \frac{|p|^{4}}{8}
$$


Hence, for all $\tau \in W^{1, \infty}(\Sigma)$,

$$
\begin{aligned}
E(\tau) & =\left(2 \int_{\Sigma}\left(\sqrt{1+\left|\nabla^{\prime} \tau\right|^{2}}-1\right) d x^{\prime}-G \int_{\Sigma} \tau^{2} d x^{\prime}\right) \\
& \geq\left(\int_{\Sigma}\left|\nabla^{\prime} \tau\right|^{2} d x^{\prime}-G \int_{\Sigma} \tau^{2} d x^{\prime}-\frac{1}{4} \int_{\Sigma}\left|\nabla^{\prime} \tau\right|^{4} d x^{\prime}\right) .
\end{aligned}
$$

Notice that from (6.13) it becomes clear that $E(\tau)$ is positive for suitably small $\left\|\nabla^{\prime} \tau\right\|_{\infty}$ if and only if its linearized part $E_{L}(\tau)$ is positive. Therefore, provided the linearized energy $E_{L}(\tau)$, namely $\int_{\Sigma}\left|\nabla^{\prime} \tau\right|^{2} d x^{\prime}-G \int_{\Sigma} \tau^{2} d x^{\prime}$, is positive also the total energy $E(\tau)$ will be positive for $\nabla^{\prime} \tau$ sufficiently small in the norm of $L^{\infty}$. Thus to completely prove Theorem 6.1 we show that $E(\tau)$ for small $\|\tau\|_{\infty}$ is still positive. For an arbitrary function $\tau$ we consider the Fourier expansion and we get again the result. By using the eigenfunction expansion in $L_{0}^{2}$, with $\gamma_{k}=\left(\tau, \tau_{k}\right), \tau$ is expressed by

$$
\tau=\sum_{k=1}^{\infty} \gamma_{k} \tau_{k}, \quad \nabla^{\prime} \tau=\sum_{k=1}^{\infty} \gamma_{k} \nabla^{\prime} \tau_{k}
$$

By (6.6) it follows that

$$
\begin{aligned}
\int_{\Sigma}\left|\nabla^{\prime} \tau\right|^{2} d x^{\prime}-G \int_{\Sigma} \tau^{2} d x^{\prime} & =\sum_{k=1}^{\infty} \gamma_{k}^{2} \int_{\Sigma}\left(\left|\nabla^{\prime} \tau_{k}\right|^{2} d x^{\prime}-G \tau_{k}^{2}\right) d x^{\prime} \\
& =\sum_{k=1}^{\infty} \gamma_{k}^{2} \lambda_{k} \int_{\Sigma} \tau_{k}^{2} d x^{\prime}=\sum_{k=1}^{\infty} \gamma_{k}^{2} \lambda_{k} .
\end{aligned}
$$

On the other hand, by the assumption $\left\|\nabla^{\prime} \tau\right\|_{\infty} \leq \delta$, we get

$$
\begin{aligned}
\int_{\Sigma}\left|\nabla^{\prime} \tau\right|^{4} d x^{\prime} & \leq \delta^{2} \int_{\Sigma}\left|\nabla^{\prime} \tau\right|^{2} d x^{\prime} \\
& =\delta^{2} \sum_{k=1}^{\infty} \gamma_{k}^{2} \int_{\Sigma}\left|\nabla^{\prime} \tau_{k}\right|^{2} d x^{\prime}=\delta^{2} \sum_{k=1}^{\infty} \gamma_{k}^{2}\left(G+\lambda_{k}\right)
\end{aligned}
$$

As a consequence it yields:

$$
\begin{aligned}
E(\tau) & \geq \sum_{k=1}^{\infty}\left(\gamma_{k}^{2} \lambda_{k}-\frac{\delta^{2}}{4} \gamma_{k}^{2}\left(G+\lambda_{k}\right)\right) \int_{\Sigma} \tau_{k}^{2} d x^{\prime} \\
& =\sum_{k=1}^{\infty} \gamma_{k}^{2}\left[\lambda_{k}\left(1-\frac{\delta^{2}}{4}\right)-\frac{\delta^{2}}{4} G\right] \int_{\Sigma} \tau_{k}^{2} d x^{\prime} .
\end{aligned}
$$

Hence, recalling that $\lambda_{1}=G_{d}-G$, if

$$
\left(G_{d}-G\right)-\frac{G_{d}}{4} \delta^{2} \geq 0
$$

namely for

$$
\delta^{2} \leq 4 \frac{\left(G_{d}-G\right)}{G_{d}}
$$

we obtain

$$
E(\tau) \geq 0
$$


Therefore we have proved that if $\tau$ satisfies (6.10) with $\delta$ bounded above by (6.15), for $\delta$ sufficiently small, the initial value $\left(\mathbf{u}_{0}, \tau\right)=(\mathbf{0}, \tau)$ is such that the energy at initial time (3.6) is positive; hence the rest state is nonlinearly stable.

Condition (6.15) states that the size of the initial data decreases as $G \rightarrow G_{d}$ and tends to zero as $G$ tends to $G_{d}$, so that the size of the initial data will no longer be controllable for $G$ too close to $G_{d}$. The interest of this formula lies in its simplicity.

Proof of Theorem 6.2: Negativeness of $E\left(\eta_{0}\right)$ for large initial data. In this part we assume $d<1$ because we wish to just give an explicit example. Here we prove that with our example it is possible to construct large initial values which furnish negative initial energy. We look for a function $\tau$ with $E(\tau)<0$, for a sufficiently large number $L=\|\tau\|_{L^{2}}$. We choose $\eta_{0}=L \tau_{1}$. Recalling (6.13), it follows that

$$
E(\tau)=\int_{\Sigma}\left(2\left(\sqrt{1+\left|\nabla^{\prime} \tau\right|^{2}}-1\right)-G \tau^{2}\right) d x^{\prime} .
$$

We must construct one initial data such that $E(\tau)<0$. Take $\tau=L \tau_{1}$, where

$$
\left\|\tau_{1}\right\|_{L^{2}(\Sigma)}^{2}=\frac{1}{\lambda_{1}+G}\left\|\nabla^{\prime} \tau_{1}\right\|_{L^{2}(\Sigma)}^{2}=\frac{1}{G_{d}}\left\|\nabla^{\prime} \tau_{1}\right\|_{L^{2}(\Sigma)}^{2}
$$

is the first eigenvalue and $L$ is the intensity of the initial data in $L^{2}$. To simplify calculations, in this computation we set

$$
L\left|\nabla^{\prime} \tau_{1}\right|=p
$$

and substitute the value of the first eigenvalue, $\lambda_{1}=G_{d}-G$,

$$
E(\tau)=\int_{\Sigma}\left(2\left(\sqrt{1+p^{2}}-1\right)-\frac{G}{G_{d}} p^{2}\right) d x^{\prime}
$$

The sign of this functional is reduced to the sign of the integrand; hence we compute the sign of the integrand. Simple calculations lead to

$$
\left(1-\frac{G}{G_{d}}\right) p^{2}-\frac{p^{2}}{\left(1+\sqrt{1+p^{2}}\right)^{2}} p^{2}=p^{2}\left\{\frac{G_{d}-G}{G_{d}}-\frac{p^{2}}{\left(1+\sqrt{1+p^{2}}\right)^{2}}\right\} .
$$

If

$$
\frac{G_{d}-G}{G_{d}} \int_{\Sigma} p^{2} d x^{\prime}-\int_{\Sigma} p^{4} \frac{1}{\left(1+\sqrt{1+p^{2}}\right)^{2}} d x^{\prime}<0
$$

then $E(\tau)<0$. To give a bound in terms of the intensity $L$ of the $L^{2}$-norm of the initial data, we consider the case $d<1$, with $\nabla^{\prime} \tau_{1}=\left(\sqrt{\frac{2}{d}} 2 \pi \cos \left(2 \pi x_{1}\right), 0\right)$, and $\tau_{1}$ having unitary $L^{2}$-norm. Therefore it follows that

$$
0<\left|\cos ^{2}\left(2 \pi x_{1}\right)\right|<1 \quad \Longleftrightarrow \quad 0<p^{2}<\frac{8 \pi^{2} L^{2}}{d} .
$$

(6.19) implies that

$$
\begin{array}{r}
-\int_{\Sigma} p^{4} \frac{1}{\left(1+\sqrt{1+p^{2}}\right)^{2}} d x^{\prime} \leq-\frac{1}{\left(1+\sqrt{1+\left(8 \pi^{2} L^{2} / d\right)}\right)^{2}} \int_{\Sigma} p^{4} d x^{\prime} \\
-\int_{\Sigma} \sin ^{4}(2 \pi x) d x^{\prime}=-\int_{\Sigma} \cos ^{4}\left(2 \pi x_{1}\right) d x^{\prime}=-\frac{3 d}{8} .
\end{array}
$$

\footnotetext{
${ }^{*}$ We recall that $d<1$ means small wetting area.
} 
Furthermore, it follows that

$$
\int_{\Sigma} p^{2} d x^{\prime}=4 \pi^{2} L^{2}
$$

From the above calculations we deduce that $E$ is negative if

$$
\frac{G_{d}-G}{G_{d}} 4 \pi^{2} L^{2}-\frac{24 \pi^{4} L^{4}}{d\left(1+\sqrt{1+\left(8 \pi^{2} L^{2} / d\right)}\right)^{2}}<0 .
$$

We set $\gamma:=\left(G_{d}-G\right) / G_{d}$ and write

$$
\gamma-\frac{6 \pi^{2} L^{2}}{d\left(1+\sqrt{1+\frac{8 \pi^{2} L^{2}}{d}}\right)^{2}}<0
$$

Developing the square we have

$$
2 \gamma d+\gamma d \frac{8 \pi^{2} L^{2}}{d}+2 \gamma d \sqrt{1+\frac{8 \pi^{2} L^{2}}{d}}-\frac{3 d}{4} \frac{8 \pi^{2} L^{2}}{d}<0 .
$$

In an equivalent way we write

$$
\left(1+\frac{8 \pi^{2} L^{2}}{d}\right)\left(\gamma-\frac{3}{4}\right)+2 \gamma \sqrt{1+\frac{8 \pi^{2} L^{2}}{d}}+2 \gamma-\left(\gamma-\frac{3}{4}\right)<0 .
$$

Setting $x:=\sqrt{1+\frac{8 \pi^{2} L^{2}}{d}}$, we get

$$
\left(\gamma-\frac{3}{4}\right) x^{2}+2 \gamma x+\left(\gamma+\frac{3}{4}\right)<0
$$

This algebraic inequality admits two resolution functions of the coefficient of $x^{2}$. We assume

$$
\left(\gamma-\frac{3}{4}\right)<0, \quad \Longleftrightarrow 4 G>G_{d}
$$

and we change all signs in the inequality, obtaining

$$
x^{2}\left(\frac{3}{4}-\gamma\right)-2 \gamma x-\left(\gamma+\frac{3}{4}\right)>0
$$

Taking the positive root it must happen that

$$
x \geq \frac{4 \gamma+3}{(3-4 \gamma)}
$$

Recall that $3-4 \gamma>0$. Substituting the values of $x$ and $\gamma$, we have

$$
\frac{8 \pi^{2}}{d} L^{2}>\left(\frac{7 G_{d}-4 G}{4 G-G_{d}}\right)^{2}-1
$$

Therefore, we have

$$
L^{2}>\frac{d}{8 \pi^{2}} \frac{48 G_{d}}{\left(4 G-G_{d}\right)^{2}}\left(G_{d}-G\right)
$$

that is,

$$
L^{2}>24 d \frac{G_{d}-G}{\left(4 G-G_{d}\right)^{2}} .
$$

For such a $\tau$, we obtain $E(\tau)<0$. Therefore we know that for initial data $\eta_{0}=L \tau_{1}$ with $L$ satisfying condition (6.26), the initial value $\left(\mathbf{u}_{0}, \eta_{0}\right)=\left(\mathbf{0}, L \tau_{1}\right)$ brings an initial negative energy $E_{0}$. 
REMARK 6.1. We read condition (6.26) as a condition on the Grashof number $G$, recalling that $G$ must belong to the interval $\left(\frac{G_{d}}{4}, G_{d}\right)$. Moreover, $G$ acts against stability; actually as $G$ goes to $G_{d} / 4, L^{2}$ must go to $\infty$, and there is instability only for initial data that are extremely large, which means never! This result seems interesting. Moreover, if $G$ goes to $G_{d}$, then the initial data may become extremely small and there is instability also for small initial data. Another factor to be analyzed is the size of the area of the contact section $|\Sigma|$ between the liquid and the plane. If $d$ goes to zero, also $L^{2}$ goes to zero; thus instability may occur for a thin contact section.

REMark 6.2. The conditions on $g_{0}, \sigma, h, d$ need to be written out. In our example, we require the following conditions:

$$
\begin{aligned}
& \frac{G_{d}}{4}<G<G_{d}, \\
& h+L \tau_{1}>\frac{h}{2}, \\
& \operatorname{vol} V=h d=1 .
\end{aligned}
$$

From the first two inequalities and (6.26), we get

$$
\frac{h}{2}+L \tau_{1}>\frac{h}{2}-L\left|\tau_{1}\right| \geq \frac{h}{2}-L \sqrt{\frac{2}{d}} .
$$

Hence the left-hand side of (6.28) will be positive if

$$
\frac{h}{2} \geq L \sqrt{\frac{2}{d}}=2 \sqrt{6 d} \frac{\sqrt{G_{d}-G}}{\left(4 G-G_{d}\right)} \sqrt{\frac{2}{d}}=4 \sqrt{3} \frac{\sqrt{G_{d}-G}}{\left(4 G-G_{d}\right)} .
$$

We may read (6.29) as a compatibility condition on the depth of the layer in the function of $G$ : for instability to occur, the depth must be sufficiently large. On the other side, from (6.27) 3 we obtain a compatibility condition on the cross section as a function of $G$; precisely,

$$
\left(4 G-G_{d}\right)>8 \sqrt{3} d \sqrt{\left(G_{d}-G\right)} .
$$

We read condition (6.30) as a condition of smallness on the area of the contact section $|\Sigma|$, the wet area. For instability to occur, the wet area must be sufficiently small.

\section{Appendix.}

7.1. Linear stability. Through the whole paper we have compared the two linear and nonlinear stability concepts for the equilibrium configuration of a liquid layer. Notice that nonlinear stability is related to the behavior in time of solutions of the full nonlinear system say to (2.16). To study this problem we have used as a global, qualitative method the Lyapunov method known as the modified energy. However, the linear stability problem has been always considered known, since it is based on spectral analysis. For the sake of completeness, in this section we give a sketch of a proof that the modified energy method also works in the linear case. 
The nonstationary system obtained as a linearization to (2.16):

$$
\begin{array}{ll}
\partial_{t} \mathbf{u}-\nabla \cdot T(\mathbf{u}, p)=g_{0} \nabla x_{3} & \text { in } \Omega_{b}, t>0, \\
\nabla \cdot \mathbf{u}=0 & \text { in } \Omega_{b}, t>0, \\
T(\mathbf{u}, p) \mathbf{e}_{3}=\sigma \Delta^{\prime} \eta \mathbf{e}_{3}-q_{e} \mathbf{e}_{3} & \text { on } \Gamma_{b}, t>0, \\
\partial_{t} \eta=u_{3} & \text { on } \Gamma_{b}, t>0, \\
\mathbf{u}=\mathbf{0} & \text { on } \Gamma_{B}, t>0, \\
\left.\mathbf{u}\right|_{t=0}=\mathbf{u}_{0} \text { in } \Omega_{b},\left.\quad \eta\right|_{t=0}=\tau \text { on } \Sigma . &
\end{array}
$$

Here all the notation of (2.16) is preserved. It is trivial that in (7.1) the domain is fixed; therefore linearized equations represent a deep simplification of the nonlinear ones! Since the domain in now fixed, we set

$$
\|\varphi\|_{L^{2}\left(\Omega_{t}\right)}^{2}=\|\varphi\|_{L^{2}\left(\Omega_{b}\right)}^{2} .
$$

We also drop the subscript $\Omega_{b}$ added to the scalar product $($,$) in L^{2}$. It is not difficult to prove that the energy equation holds.

Theorem 7.1. Let $(\mathbf{u}, \eta, p) \in \mathcal{W}$ satisfy (7.1). Then it follows that

$$
\frac{d}{d t}\left[-g_{0}\|\eta\|_{L^{2}(\Sigma)}^{2}+\sigma\left\|\nabla^{\prime} \eta\right\|_{L^{2}(\Sigma)}^{2}+\|\mathbf{u}\|_{L^{2}\left(\Omega_{b}\right)}^{2}\right]+\mu\|S(\mathbf{u})\|_{L^{2}\left(\Omega_{b}\right)}^{2}=0 .
$$

Proof. Multiplying (7.1) by $\mathbf{u}$ and integrating over $\Omega_{b}$, and integrating by parts we easily obtain the energy equation (7.2).

We set

$$
\mathcal{E}_{L}(\mathbf{u}, \eta)=-g_{0}\|\eta\|_{L^{2}(\Sigma)}^{2}+\sigma\left\|\nabla^{\prime} \eta\right\|_{L^{2}(\Sigma)}^{2}+\|\mathbf{u}\|_{L^{2}\left(\Omega_{b}\right)}^{2} .
$$

We now study the exponential decay of solutions $(\mathbf{u}, p, \eta) \in \mathcal{W}$ to (7.1) by the energy method.

Theorem 7.2. We assume that the linear stability hypothesis (6.9):

$$
G<G_{d}:=4 \pi^{2} \min \left\{1, \frac{1}{d^{2}}\right\}
$$

holds. Assume that there exist solutions $(\mathbf{u}, \eta, p)$ to (7.1) with corresponding initial data $\left(\mathbf{u}_{0}, \eta_{0}\right)$. In this case the initial data $\left(\mathbf{u}_{0}, \eta_{0}\right) \in L^{2}\left(\Omega_{b}\right) \times W^{1, \infty}(\Sigma)$ has positive initial energy:

$$
\mathcal{E}_{L}\left(\mathbf{u}_{0}, \eta_{0}\right)=-g_{0}\left\|\eta_{0}\right\|_{L^{2}(\Sigma)}^{2}+\sigma\left\|\nabla^{\prime} \eta_{0}\right\|_{L^{2}(\Sigma)}^{2}+\left\|\mathbf{u}_{0}\right\|_{L^{2}\left(\Omega_{b}\right)}^{2}>0
$$

because $\mathcal{E}_{L}\left(\mathbf{u}_{0}, \eta_{0}\right)$ is a positive definite quadratic form in the variables $\left\|\nabla^{\prime} \eta_{0}\right\|_{L^{2}(\Sigma)}^{2}$ and $\left\|\mathbf{u}_{0}\right\|_{L^{2}\left(\Omega_{b}\right)}^{2}$. Then the rest state $S_{b}$ is linearly stable; namely, there exists a constant $b>0$ such that

$$
\mathcal{E}_{L}(\mathbf{u}, \eta)(t)<C \mathcal{E}_{L}\left(\mathbf{u}_{0}, \eta_{0}\right) \exp ^{-b t}, \quad \forall t>0
$$

holds.

Proof. Let us multiply (7.1) by $\mathbf{V}$ given in Lemma 7.3 with $\Omega_{t} \rightarrow \Omega_{b}, \Gamma_{t}=\Gamma_{b}$ and integrate over $\Omega_{b}$. This gives

$$
\frac{d}{d t}(\mathbf{u}, \mathbf{V})_{\Omega_{b}}+\int_{\Gamma_{b}}\left(-\sigma \Delta^{\prime} \eta-g_{0} \eta\right) \mathbf{V} \cdot \mathbf{e}_{3} d x^{\prime}=\mathcal{F}(\mathbf{u}, \mathbf{V})
$$


where

$$
\mathcal{F}(\mathbf{u}, \mathbf{V})=\left(\mathbf{u}, \partial_{t} \mathbf{V}\right)_{\Omega_{b}}-\mu(S(\mathbf{u}), \nabla \mathbf{V})_{\Omega_{b}} .
$$

Integrating by parts in the surface integral in (7.4), and recalling the boundary value of $\mathbf{V}$ yields

$$
\frac{d}{d t}(\mathbf{u}, \mathbf{V})_{\Omega_{b}}+\int_{\Sigma}\left(\sigma\left|\nabla^{\prime} \eta\right|^{2}-g_{0}|\eta|^{2}\right) d x^{\prime}=\mathcal{F}(\mathbf{u}, \mathbf{V})
$$

Adding (7.2) to (17.6) multiplied by a positive constant $\gamma$ yields

$$
\begin{aligned}
\frac{d}{d t}\left[-g_{0}\|\eta\|_{L^{2}(\Sigma)}^{2}+\right. & \left.\sigma\left\|\nabla^{\prime} \eta\right\|_{L^{2}(\Sigma)}^{2}+\|\mathbf{u}\|_{L^{2}\left(\Omega_{b}\right)}^{2}+\gamma(\mathbf{u}, \mathbf{V})_{\Omega_{t}}\right] \\
& +\mu\|S(\mathbf{u})\|_{L^{2}\left(\Omega_{b}\right)}^{2}+\gamma \int_{\Sigma}\left(\sigma\left|\nabla^{\prime} \eta\right|^{2}-g_{0}|\eta|^{2}\right) d x^{\prime}=\gamma \mathcal{F}(\mathbf{u}, \mathbf{V}) .
\end{aligned}
$$

We introduce the modified energy

$$
\mathbb{E}=-g_{0}\|\eta\|_{L^{2}(\Sigma)}^{2}+\sigma\left\|\nabla^{\prime} \eta\right\|_{L^{2}(\Sigma)}^{2}+\|\mathbf{u}\|_{L^{2}\left(\Omega_{b}\right)}^{2}+\gamma(\mathbf{u}, \mathbf{V})_{\Omega_{b}} .
$$

By (2.1), (5.21) and Lemma 7.3, we obtain

$$
\begin{aligned}
\mathbb{E} & \leq \sigma\left\|\nabla^{\prime} \eta\right\|_{L^{2}(\Sigma)}^{2}+\|\mathbf{u}\|_{L^{2}\left(\Omega_{b}\right)}^{2}+\frac{\gamma}{2}\left(\|\mathbf{u}\|_{L^{2}\left(\Omega_{b}\right)}^{2}+\|\eta\|_{L^{2}(\Sigma)}^{2}\right) \\
& \leq \max \left\{\left(\sigma+\frac{\gamma}{2} C_{d}\right), C\left(1+\frac{\gamma}{2}\right)\right\}\left(\|S(\mathbf{u})\|_{L^{2}\left(\Omega_{b}\right)}^{2}+\left\|\nabla^{\prime} \eta\right\|_{L^{2}(\Sigma)}^{2}\right) .
\end{aligned}
$$

Below, we prove that there exists a positive constant $\beta$ which satisfies

$$
\begin{aligned}
& \mu\|S(\mathbf{u})\|_{L^{2}\left(\Omega_{b}\right)}^{2}+\gamma \int_{\Sigma}\left(\sigma\left|\nabla^{\prime} \eta\right|^{2}-g_{0}|\eta|^{2}\right) d x^{\prime}-\gamma \mathcal{F}(\mathbf{u}, \mathbf{V}) \\
& \quad \geq \beta\left(\|S(\mathbf{u})\|_{L^{2}\left(\Omega_{b}\right)}^{2}+\left\|\nabla^{\prime} \eta\right\|_{L^{2}(\Sigma)}^{2}\right) .
\end{aligned}
$$

By (2.1) with $C_{d}=1 / G_{d}$ we have

$$
\int_{\Sigma}\left(\sigma\left|\nabla^{\prime} \eta\right|^{2}-g_{0}|\eta|^{2}\right) d x^{\prime} \geq\left(\sigma-\frac{g_{0}|\Sigma|}{G_{d}}\right) \int_{\Sigma}\left|\nabla^{\prime} \eta\right|^{2} d x^{\prime}
$$

where $\sigma-\left(g_{0}|\Sigma|\right) / G_{d}>0$ because of the linear stability assumption: $G<G_{d}$. By (5.25),

$$
|\mathcal{F}(\mathbf{u}, \mathbf{V})| \leq C\|S(\mathbf{u})\|_{L^{2}\left(\Omega_{b}\right)}^{2}+c \mu\|S(\mathbf{u})\|_{L^{2}\left(\Omega_{b}\right)}\left\|\nabla^{\prime} \eta\right\|_{L^{2}(\Sigma)} .
$$

By (7.11), (7.12) we have

$$
\begin{gathered}
\mu\|S(\mathbf{u})\|_{L^{2}\left(\Omega_{b}\right)}^{2}+\gamma \int_{\Sigma}\left(\sigma\left|\nabla^{\prime} \eta\right|^{2}-g_{0}|\eta|^{2}\right) d x^{\prime}-\gamma \mathcal{F}(\mathbf{u}, \mathbf{V}) \\
\geq \mu\|S(\mathbf{u})\|_{L^{2}\left(\Omega_{b}\right)}^{2}+\gamma\left(\sigma-\frac{g_{0}|\Sigma|}{G_{d}}\right)\left\|\nabla^{\prime} \eta\right\|_{L^{2}(\Sigma)}^{2} \\
-\gamma C\|S(\mathbf{u})\|_{L^{2}\left(\Omega_{b}\right)}^{2}-\gamma c \mu\|S(\mathbf{u})\|_{L^{2}\left(\Omega_{b}\right)}\left\|\nabla^{\prime} \eta\right\|_{L^{2}(\Sigma)} .
\end{gathered}
$$

From the inequality

$$
\gamma c \mu\|S(\mathbf{u})\|_{L^{2}\left(\Omega_{b}\right)}\left\|\nabla^{\prime} \eta\right\|_{L^{2}(\Sigma)} \leq \gamma c\left(\frac{\mu^{2} G_{d}}{G_{d} \sigma-g_{0}|\Sigma|}\|S(\mathbf{u})\|_{L^{2}\left(\Omega_{b}\right)}^{2}+\frac{G_{d} \sigma-g_{0}|\Sigma|}{4 G_{d}}\left\|\nabla^{\prime} \eta\right\|_{L^{2}(\Sigma)}^{2}\right)
$$


and (7.13), it follows that

$$
\begin{aligned}
& \mu\|S(\mathbf{u})\|_{L^{2}\left(\Omega_{b}\right)}^{2}+\gamma \int_{\Sigma}\left(\sigma\left|\nabla^{\prime} \eta\right|^{2}-g_{0}|\eta|^{2}\right) d x^{\prime}-\gamma \mathcal{F}(\mathbf{u}, \mathbf{V}) \\
\geq & \left\{\mu-\gamma\left(C+\frac{c \mu^{2} G_{d}}{G_{d} \sigma-g_{0}|\Sigma|}\right)\right\}\|S(\mathbf{u})\|_{L^{2}\left(\Omega_{b}\right)}^{2}+3 \gamma \frac{G_{d} \sigma-g_{0}|\Sigma|}{4 G_{d}}\left\|\nabla^{\prime} \eta\right\|_{L^{2}(\Sigma)}^{2} .
\end{aligned}
$$

If we choose $\gamma$ so small such that

$$
\mu-\gamma\left(C+\frac{c \mu^{2} G_{d}}{G_{d} \sigma-g_{0}|\Sigma|}\right)>0
$$

then we obtain (7.10). Therefore combining

$$
\frac{d}{d t} \mathbb{E}+\beta\left(\|S(\mathbf{u})\|_{L^{2}\left(\Omega_{b}\right)}^{2}+\left\|\nabla^{\prime} \eta\right\|_{L^{2}(\Sigma)}^{2}\right) \leq 0
$$

and (7.9), we obtain

$$
\frac{d}{d t} \mathbb{E}+b \mathbb{E} \leq 0, \quad b=\frac{\beta}{\max \left\{\left(\sigma+\frac{\gamma}{2} C_{d}\right), C\left(1+\frac{\gamma}{2}\right)\right\}} .
$$

Therefore we obtain

$$
\mathbb{E} \leq \mathbb{E}_{0} \exp ^{-b t}
$$

with

$$
\mathbb{E}_{0}=-g_{0}\left\|\eta_{0}\right\|_{L^{2}(\Sigma)}^{2}+\sigma\left\|\nabla^{\prime} \eta_{0}\right\|_{L^{2}(\Sigma)}^{2}+\left\|\mathbf{u}_{0}\right\|_{L^{2}\left(\Omega_{b}\right)}^{2}+\gamma \int_{\Omega_{b}} \mathbf{u}_{03} \eta_{0} d x
$$

where we have used $\mathbf{V}(x, 0)=\left(0,0, \eta_{0} \chi\left(x_{3}\right)\right)$ (cf. Lemma 7.4). Since $\mathcal{E}_{L}$ and $\mathbb{E}$ are equivalent because of the inequalities

$$
\begin{aligned}
& \left(\sigma-\frac{g_{0}|\Sigma|}{G_{d}}\right)\left\|\nabla^{\prime} \eta_{0}\right\|_{L^{2}(\Sigma)}^{2}+\left\|\mathbf{u}_{0}\right\|_{L^{2}\left(\Omega_{b}\right)}^{2} \leq \mathcal{E}_{L} \leq \sigma\left\|\nabla^{\prime} \eta_{0}\right\|_{L^{2}(\Sigma)}^{2}+\left\|\mathbf{u}_{0}\right\|_{L^{2}\left(\Omega_{b}\right)}^{2}, \\
& \left(\sigma-\frac{g_{0}|\Sigma|}{G_{d}}-\frac{\gamma}{2 G_{d}}\right)\left\|\nabla^{\prime} \eta_{0}\right\|_{L^{2}(\Sigma)}^{2}+\left(1-\frac{\gamma}{2}\right)\left\|\mathbf{u}_{0}\right\|_{L^{2}\left(\Omega_{b}\right)}^{2} \\
& \quad \leq \mathbb{E} \leq\left(\sigma+\frac{\gamma}{2 G_{d}}\right)\left\|\nabla^{\prime} \eta_{0}\right\|_{L^{2}(\Sigma)}^{2}+\left(1+\frac{\gamma}{2}\right)\left\|\mathbf{u}_{0}\right\|_{L^{2}\left(\Omega_{b}\right)}^{2},
\end{aligned}
$$

if we take $\gamma>0$ so small such that

$$
\sigma-\frac{g_{0}|\Sigma|}{G_{d}}-\frac{\gamma}{2 G_{d}}>0
$$

we obtain

$$
\mathcal{E}_{L}(\mathbf{u}, \eta)(t)<C \mathcal{E}_{L}\left(\mathbf{u}_{0}, \eta_{0}\right) \exp ^{-b t}, \quad \forall t>0
$$


7.2. Auxiliary function. In this subsection we construct the auxiliary function employed in the free work equation.

Lemma 7.3 (Lemma 2.1 in [20]). Let there be given the field $\eta \in L^{\infty}\left(0, \infty ; W_{\natural}^{1, \infty}(\Sigma)\right.$ ) with $\partial_{t} \eta \in L^{\infty}\left(0, \infty ; L_{\natural}^{2}(\Sigma)\right)$. Then there exists a vector field $\mathbf{V} \in L^{\infty}\left(0, \infty ; W_{\natural}^{1,2}\left(\Omega_{t}\right)\right)$ with $\partial_{t} \mathbf{V} \in L^{\infty}\left(0, \infty ; L_{\natural}^{2}\left(\Omega_{t}\right)\right)$ which solves the following problem:

$$
\begin{aligned}
\nabla \cdot \mathbf{V}=0 & \text { in } \Omega_{t}, \\
\mathbf{V}=\mathbf{0} & \text { on } \Gamma_{B}, \\
\mathbf{V} \cdot \tilde{\mathbf{n}}=\eta & \text { on } \Gamma_{t},
\end{aligned}
$$

and satisfies the estimates

$$
\begin{aligned}
\left\|\partial_{t} \mathbf{V}\right\|_{L^{2}\left(\Omega_{t}\right)} & \leq C\|\nabla \mathbf{u}\|_{L^{2}\left(\Omega_{t}\right)}, \\
\|\nabla \mathbf{V}\|_{L^{2}\left(\Omega_{t}\right)} & \leq C\left\|\nabla^{\prime} \eta\right\|_{L^{2}(\Sigma)}, \\
\|\mathbf{V}\|_{L^{2}\left(\Omega_{t}\right)} & \leq C\|\eta\|_{W^{1,2}(\Sigma)}
\end{aligned}
$$

with positive constants $C$.

Proof. We prove the lemma by following the proof of Lemma 2.1 in 20. Since the condition (2.20) is satisfied, the periodic solution in $\Sigma$ of the Poisson equation for $\eta \in$ $L^{\infty}\left(0, \infty ; W_{\natural}^{1, \infty}(\Sigma)\right)$,

$$
\Delta^{\prime} \varphi=\eta, \quad x^{\prime} \in \mathbb{R}_{\natural}^{2},
$$

is unique (up to a constant). We set

$$
\begin{gathered}
\mathbf{b}\left(x^{\prime}, t\right)=\nabla^{\prime} \varphi\left(x^{\prime}, t\right), \\
\mathbf{A}\left(x^{\prime}, t\right)=\left(-b_{2}\left(x^{\prime}, t\right), b_{1}\left(x^{\prime}, t\right), 0\right) .
\end{gathered}
$$

We look for a solution in the form

$$
\mathbf{V}=\nabla \times\left(\mathbf{A}\left(x^{\prime}, t\right) \chi\left(x_{3}\right)\right),
$$

where $\chi\left(x_{3}\right) \in C^{\infty}$ is a cutoff function $\chi\left(x_{3}\right)=1$ for $x_{3} \geq h+\inf _{t>0} \eta\left(x^{\prime}, t\right)$ and $\chi\left(x_{3}\right)=0$ for $x_{3} \leq f\left(x^{\prime}\right)$ (cf. (2.2) $)$. Since

$$
\nabla \times \mathbf{A} \cdot \mathbf{n}=n_{1}\left(\frac{\partial A_{3}}{\partial x_{2}}-\frac{\partial A_{2}}{\partial x_{3}}\right)+n_{2}\left(\frac{\partial A_{1}}{\partial x_{3}}-\frac{\partial A_{3}}{\partial x_{1}}\right)+n_{3}\left(\frac{\partial A_{2}}{\partial x_{1}}-\frac{\partial A_{1}}{\partial x_{3}}\right)
$$

and

$$
\begin{aligned}
& \frac{\partial A_{3}}{\partial x_{2}}-\frac{\partial A_{2}}{\partial x_{3}}=0, \quad \frac{\partial A_{1}}{\partial x_{3}}-\frac{\partial A_{3}}{\partial x_{1}}=0, \\
& \frac{\partial A_{2}}{\partial x_{1}}-\frac{\partial A_{1}}{\partial x_{3}}=\frac{\partial b_{1}}{\partial x_{1}}+\frac{\partial b_{2}}{\partial x_{2}}=\nabla^{\prime} \cdot \mathbf{b}=\Delta \varphi=\eta,
\end{aligned}
$$

we have

$$
\left.\mathbf{V} \cdot \mathbf{n}\right|_{\Gamma}=n_{3} \eta\left(x^{\prime}, t\right) .
$$


By the definition of $\mathbf{V}$, we easily obtain

$$
\begin{gathered}
\left.\mathbf{V}(x, t)\right|_{\Gamma_{B}}=\left.\mathbf{V}(x, t)\right|_{x_{3}=f\left(x^{\prime}\right)}=\mathbf{0}, \\
\nabla \cdot \mathbf{V}=0 \text { in } \Omega_{t}, \\
\mathbf{V}(x, t)=\left(0,0, \eta\left(x^{\prime}, t\right) \chi\left(x_{3}\right)\right)
\end{gathered}
$$

and the desired estimates (7.16)-(7.18), because $L^{\infty}(\Sigma) \subset L^{2}(\Sigma)$.

Acknowledgments. Padula thanks $60 \%$ MURST, the GNFM of the Italian CNRINDAM, and 40\% MIUR-COFIN 2006-2008 "La matematica dei processi di crescita tumorali e trasporto nelle applicazioni biomediche e industriali". One author thanks Profs. A. Petrovna and V.A. Solonnikov for stimulating discussions on the subject. The work of Shimizu is partially supported by JSPS Grant-in-aid for Scientific Research C\#20540164.

\section{REFERENCES}

[1] H. Abels, The initial-value problem for the Navier-Stokes equations with a free surface in $L_{q}$ Sobolev spaces, Adv. Differential Equations, 10 (2005), 45-64. MR2106120 (2005j:35164)

[2] G. Allain, Small-time existence for the Navier-Stokes equations with a free surface, Appl. Math. Optim., 16 (1987), 37-50. MR883473 (88e:35147)

[3] J. T. Beale, Large-time behavior of viscous surface waves, Arch. Rational Mech. Anal., 84 (1984), 307-352. MR 721189 (85c:35074)

[4] J. T. Beale and T. Nishida, Large-time behaviour of viscous surface waves, Lecture Notes in Num. Appl. Anal., 8 (1985), 1-14. MR882925 (88f:35121)

[5] S. Chandrasekhar, Hydrodynamic and hydromagnetic stability, Dover Pub. Inc., New York, 1981.

[6] P. G. Drazin and W. H. Reid, Hydrodynamic stability, Cambridge Univ. Press, 1981. MR604359 (82h:76021)

[7] R. Finn, On equations of capillarity, J. Math. Fluid Mech. 3 (2001), 139-151. MR1838954 (2002c:76026)

[8] E. Frolova and M. Padula, Free boundary problem for a layer of inhomogeneous fluid, European J. Mech. B/Fluids, 23 (2004), 665-679. MR2148676 (2006a:76030)

[9] G. P. Galdi and M. Padula, A new approach to energy theory in the stability of fluid motion, Arch. Rational. Mech. Anal. 110 (1990), 187-197. MR.1060804 (92a:76050)

[10] B.J. Jin and M. Padula, On the existence of compressible viscous flow in a horizontal layer with upper free surface, Commun. Pure Appl. Anal. 1 (2002), 379-415. MR1903004(2003i:35285)

[11] B.J. Jin and M. Padula, Steady flows of compressible fluids in a rigid container with upper free boundary, Math. Ann. 329 (2004), 723-770. MR2076684 (2005e:35188)

[12] B. Helffer and O. Lafitte, Asymptotic methods for the eigenvalues of the Rayleigh equation for linearized Rayleigh-Taylor instability, Univ. Paris-Sud. Math. 2424 (2002), 1-49. MR.1981886 (2004g:76070)

[13] G. Iooss, and M. Rossi, Nonlinear evolution of the bidimensional Rayleigh-Taylor flow, Europ. J. Mech. B/Fluids 8, 1989, 1, 1-22. MR987544(90c:76072)

[14] T. Nishida, Y. Teramoto and H. Yoshihara, Global in time behavior of viscous surface waves: horizontally periodic motion, J. Math. Kyoto Univ., 44 (2004), 271-323. MR2081074 (2005f:35246)

[15] T. Nishida, Y. Teramoto and H. Yoshihara, Hopf bifurcation in viscous incompressible flow down an inclined plane, J. Math. Fluid Mech., 7 (2005), 29-71. MR.2127741 (2005m:76049)

[16] T. Nishida, Y. Teramoto and H. A. Win, Navier-Stokes flow down an inclined plane: downward periodic motion, J. Math. Kyoto Univ., 33 (1993), 787-801. MR.1239093 (95f:35198)

[17] M. Padula, Free work and control of equilibrium configurations, Annali Università di Ferrara sez.VII, 49 (2003), 375-396. MR2165004 (2006c:74002)

[18] M. Padula, On direct Lyapunov method in continuum theories, Nonlinear problems in mathematical physics and related topics I, in honor of Prof. Ladyzhenskaya, eds. Sh. Birman, S. Hildebrandt, V.A. Solonnikov, O.A. Uralsteva, Kluwer Academic/Plenum Publisher, New York, Boston, Dordrecht, London, Moscow, 2001. MR.1970617 (2004c:76071) 
[19] M. Padula, Free Work Identity and Nonlinear Instability in Fluids with Free Boundaries, Recent Advances in Elliptic and Parabolic Problems, Proceedings of the International Conference, Hsinchu, Taiwan University, C-C Chen \& M. Chipot \& C.S. Lin, 16-20 February 2004, World Sci. (2005), 203-214. MR.2172576 (2006e:76072)

[20] M. Padula and V.A. Solonnikov, On Rayleigh-Taylor stability, Ann. Univ. Ferrara-Sez. VII - Sc. Mat. XLVI, (2000), 307-336. MR.1896938 (2003f:76052)

[21] M. Padula and V. A. Solonnikov, A simple proof of linear instability of rotating liquid drops, Annali dell'Università di Ferrara, 54 (2008), 107-122. MR2403377 (2009f:76061)

[22] J. Prüss and G. Simonett, On the two-phase Navier-Stokes equations with surface tension, in Proc. Banach Center Publication, vol. 86, Warsaw, 2009. MR2571494(2011a:35392)

[23] M. Padula and V.A. Solonnikov, Existence of non-steady flows of an incompressible viscous drop of fluid in a frame rotating with finite angular velocity, Elliptic and Parabolic Problems, Proc. of IV European Conference, World Sci. (2002), 180-203. MR1937540 (2003h:35210)

[24] V.A. Solonnikov, On the stability of axisymmetric equilibrium figures of rotating viscous incompressible liquid, Algebra Anal. 16 (2005), no. 2, 377-400. MR2068344(2005d:76016)

[25] V.A. Solonnikov, On the stability of nonsymmetric equilibrium figures of rotating viscous incompressible liquid, Interfaces and free boundaries 6 (2004), 461-492. MR2111566 (2005h:76032)

[26] V.A. Solonnikov, On instability of axially symmetric equilibrium figures of rotating viscous incompressible liquid, Zap. Nauchn. Sem. POMI 318 (2004), 277-297. MR2120803 (2006a:35257)

[27] V.A. Solonnikov, On instability of equilibrium figures of rotating viscous incompressible liquid, J. Math. Sci. 128 (2005), 3241-3262. MR2171601 (2006g:76117)

[28] V.A. Solonnikov, Letter to the editor, J. Math. Sci. 135, No. 6 (2006), 3522-3528. MR2235987 (2008b:76196)

[29] Y. Shibata and S. Shimizu, Local solvability of free surface problems for the Navier-Stokes equations with surface tension, Preprint (2008).

[30] D. Sylvester, Large time existence of small viscous surface waves without surface tension, Commun. Partial Differential Equations, 15 (1990), 823-903. MR:1070233 (91f:35216)

[31] A. Tani, Small-time existence for the three-dimensional incompressible Navier-Stokes equations with a free surface, Arch. Rat. Mech. Anal., 133 (1996), 299-331. MR.1389902 (97c:35162)

[32] A. Tani and N. Tanaka, Large time existence of surface waves in incompressible viscous fluids with or without surface tension, Arch. Rat. Mech. Anal., 130 (1995), 303-314. MR1346360 (96i:35103)

[33] Y. Teramoto, The initial value problem for a viscous incompressible flow down an inclined plane, Hiroshima Math. J., 15 (1985), 619-643. MR813577 (87b:76060)

[34] Y. Teramoto, On the Navier-Stokes flow down an inclined plane, J. Math. Kyoto Univ., 32 (1992), 593-619. MR.1183369 (93h:35162)

[35] J. A. Whitehead and M. M. Chen, Thermal instability and convection of a thin fluid layer bounded by a stably stratified region, J. Fluid Mech. 40 (1970), 549-576.

[36] M. Padula, Asymptotic stability of steady compressible flows, Lecture Notes in Mathematics (2011), to appear.

[37] M. Padula and V. A. Solonnikov, On the local solvability of free boundary problem for the NavierStokes equations, J. Math. Sci. 170 (4), 2010, 522. 